\title{
Interpolation of sublinear operators on generalized Orlicz and Hardy-Orliez spaces
}

\author{
by
}

WILLIAM T. K RA Y NEK (Pittsburgh)

\begin{abstract}
The Riesz-Thorin interpolation theorem is proved for sublinear operators on certain generalized Orlicz spaces, $L^{\psi}$. The corresponding interpolation 'theorem for the Hardy-Orlicz spaces, $H^{\psi}$, is also obtained. The interpolation theory above is extended to the case when there are certain factors, resembling the RandonNikodym derivative of measures, included. This treatment includes the known work in change of measures in the $L^{p}$-theory and generalizes the work to certain Orlicz spaces. Finally, the interpolation of $\left\{T_{z}\right\}$, a family of operators which depend on a smooth parameter, is obtained.
\end{abstract}

\section{INTRODUCTION}

Let $L^{p_{i}}, L^{q_{i}}(i=1,2)$ be Lebesgue spaces on a measure space. Let $T: L^{p_{i}} \rightarrow L^{q_{i}}$ be a linear operator such that $\|T f\|_{q_{i}} \leqslant M_{i}\|f\|_{p_{i}}, f \epsilon L^{p_{i}}$, If $p_{t}^{-1}=(1-t) p_{1}^{-1}+t p_{2}^{-1}$ and $q_{t}^{-1}=(1-t) q_{1}^{-1}+t q_{2}^{-1}$, then by the classical Riesz-Thorin interpolation theorem $T: L^{p_{t}} \rightarrow L^{q_{t}}$ such that

$$
\|T f\|_{q_{i}} \leqslant M_{1}^{1-t} M_{2}^{t}\|f\|_{p_{i}} .
$$

The importance of this result in analysis (both classical and abstract) is well-known.

In many problems of Fourier analysis, an operator $T$ is defined on the spaces above that is not linear. It is sublinear instead, i.e., it satisfies

(i) $T\left(f_{1}+f_{2}\right)$ is defined whenever $T f_{i}$ are defined,

(ii) $\left|T\left(f_{3}+f_{2}\right)\right| \leqslant\left|T f_{1}\right|+\left|T f_{2}\right|$,

(iii) $|T(a f)|=|a||T f|$, for every scalar $a$.

Calderón and Zygmund [6] were the first to treat the interpolation of sublinear operators.

Numerous other generalizations have been obtained. Stein and Weiss [30] have extended the result when the underlying measures are varied with the spaces, and Stein [29] proved an interpolation theorem for operators $T$ depending on a complex parameter $z$. Riordan [28], has 
extended Marcinkiewicz's result to Orlicz spaces, and the Riesz-Thorin theorem was extended to these spaces by Rao in (24).

Recently work on Hardy spaces was also of interest and the interpolation problem was considered there (cf. [5] and [32]). Since there is a close relation between these spaces and the Lebesgue (Orlicz) spaces, the problem is considered to include both types of spaces.

In this paper, the interpolation problem for sublinear operators is considered for certain generalized Orlicz spaces and these results are then used to obtain similar results on Hardy-Orlicz spaces. When specialized to the Lebesgue case, the $L^{p}$ spaces for $0<p \leqslant \infty$ are included, and they apply to the $H^{p}$ spaces, $0<p \leqslant \infty$, as well. Moreover, the study is always made in the case of sublinear operators. Most of the above mentioned results are subsumed in this study.

In Section 2, the interpolation theorem for sublinear operations on generalized Orlicz spaces, $L^{p}$, is proved and then, using this, the corresponding interpolation theorem for the Hardy-Orlici spaces, $H^{\varphi}$, is obtained.

In Section 3, the interpolation theory of the preceeding section is extended to the case when there are certain factors, resembling the Radon-Nikodym derivatives of measures, included. This treatment includes the known work on the change of measures in the $L^{p}$-theory and generalizes the work to certain Orlicz spaces.

Finally Section 4 contains the interpolation of $\left[T_{z}\right]$, a family of operators which depend on a smooth complex parameter. This generalizes the analytic parameter case of Stein [29]. Also the relation between interpolation with factors and change of measures is discussed here.

Generally the notation used is from [8] and [34]. Also Theorem. (Lemma and Corollary) 1.2.3 will mean Theorem (Lemma or Corollary) 3 of subsection 2 in Section 1. Similarly equation (2.1.25) will mean equation 25 of subsection 1 in Section 2.

\section{PRELIMINARIES}

In this section generalized Orlicz and Hardy spaces are defined. and some needed properties of these spaces will be given. Also some results on subharmonic functions are included for use later.

1.1. Generalized Orlicz spaces. The following results are from [18]. Generalized Young's functions, called $\varphi$-functions are needed and are given by the following:

Definterion 1.1.1. A function $\varphi(\cdot)$ is called a $\varphi$-function if $\varphi$ is continuous, defined for $u \geqslant 0$ non-decreasing, vanishing only at $u=0$, and such that $\varphi(u) \rightarrow \infty$ as $u \rightarrow \infty$.
Let $\varphi$ be a $\varphi$-function and $(\Omega, \Sigma, \mu)$ a measure space. The class of scalar valued functions $f$ measurable on $(\Omega, \Sigma, \mu)$ such that $\int_{\Omega} \varphi(|f|) d \mu<\infty$ is denoted by $L^{* \varphi}(\Omega, \Sigma, \mu)=L^{* \varphi}$ if the measure space is understood. The class $L^{\varphi}(\Omega, \Sigma, \mu)$ is the class of all (equivalence classes of) $f$ such that $\lambda f \in L^{*}(\Omega, \Sigma, \mu)$ for some $\lambda>0$. The class $L^{* \varphi}$ is a lattice and the class $L^{\varphi}$ is linear. If $\varphi$ is also convex, then $L^{\varphi}$ is called an Orlicz space on $(\Omega, \Sigma, \mu)$.

Proposition 1.1.1. A necessary and sufficient condition for $L^{* \phi}=L^{\varphi}$ is that $\varphi$ satisfy

$$
\varphi(2 u) \leqslant k p(u) \quad \text { for } u \geqslant 0 .
$$

For a proof of this proposition, see [31].

The condition (1.1.1) is called the $\Delta_{2}$-condition. The notation $\varphi \epsilon \Delta_{2}$ will mean $\varphi$ satisfies (1.1.1).

Remark. If $\varphi \in \Delta_{2}$ then it is clear that for every $k>0, \varphi(k u) \leqslant C_{k} \varphi(u)$ for $u \geqslant 0$, where $C_{k}$ depends only on $k$.

DEFINITION 1.1.2. A real valued non-negative function $\|\cdot\|$ on a linear space $X$ is called a $F$-norm lif it satisfies the following conditions

$$
\begin{aligned}
& \|x+y\| \leqslant\|x\|+\|y\| \text { for all } x, y \in X, \\
& \|x\|=0 \quad \text { if and only if } x=0 .
\end{aligned}
$$

An $F$-norm can be introduced on $L^{\varphi}$ in such a way that convergence of a sequence, $f_{n}$, to 0 with respect to this norm implies $\int_{\Omega} \varphi\left(\left|f_{n}\right|\right) d \mu \rightarrow 0$, too. This norm (throughout the paper norm will actually mean $F$-norm) is defined by:

$$
\|f\|_{\varphi}=\inf \left\{\varepsilon>0: \int_{\Omega} \varphi\left(\frac{|f|}{\varepsilon}\right) d \mu \leqslant \varepsilon\right\}
$$

and called the norm generated by $\varphi$. With this norm $L^{\varphi}(\Omega, \Sigma, \mu)$ becomes a Fréchet space, and we call $\left[L^{\varphi}(\Omega, \Sigma, \mu),\|\cdot\|_{\varphi}\right]$ a generalized Orlicz space.

Proposition 1.1.2. If $\varphi \in \Delta_{2}$, then simple functions on $(\Omega, \Sigma, \mu)$, denoted by $\mathscr{L}(\Omega, \Sigma, \mu)$, are dense in $L^{\varphi}(\Omega, \Sigma, \mu)$.

Proposirion 1.1.3. If $\varphi \in \Delta_{2}$ and $f_{n} \epsilon L_{\varphi}$ such that $\int_{\Omega} \varphi\left(\left|f_{n}\right|\right) d \mu \rightarrow 0$, then $\left\|f_{n}\right\|_{\varphi} \rightarrow 0$. define;

If $\varphi(u)=\psi\left(u^{r}\right)$ where $0<r \leqslant 1$, and $\psi$ is a convex $\varphi$-function,

$$
\|f\|_{r \varphi}=\inf \left\{\varepsilon>0: \int_{\Omega} \varphi\left(\frac{|f|}{\varepsilon^{1 / r}}\right) d \mu \leqslant 1\right\}
$$

for $f \in L^{\varphi}(\Omega, \Sigma, \mu)$. Then $\|\cdot\|_{r \varphi}$ is an $r$-homogeneous $F$-norm, that is an $F$-norm with the additional property

(1.1.5) $\quad\|a f\|_{r \varphi}=|a|^{r}\|f\|_{r \varphi} \quad$ for every scalar $a$. 
Remark. If $r=1$, then $\|\cdot\|_{1 q}$ is the Minkowski norm on $L^{\varphi}$. ([31]).

Proposition 1.1.4. The Fr-norm $\|\cdot\|_{p}$ is equivalent to $\|\cdot\|_{r \varphi}$.

1.2. Hardy-Orlicz spaces. The following results from [16] are needed for later work.

DEFINITION 1.2.1. A $\varphi$-function $\varphi$ which can be represented in the form $\varphi(u)=\Phi(\log u)$ for $u>0$ where $\Phi$ is convex on the whole axis and which satisfies $\lim _{u \rightarrow \infty} \frac{\Phi(u)}{u}=\infty$, will be called a log-convex $p$-function. Every function $\varphi$ such that $\varphi(u)=\psi\left(u^{s}\right), 0<s \leqslant 1$ and $\psi$ convex is a log-convex $p$-function since $\varphi(u)=\Phi(\log u)$ where $\Phi(u)=e^{s u}$. In the following, all $\phi$-functions will be log-convex.

Let $N$ denote the class of functions, $F$, analytic in the disk $\{z:|z|<1\}$ such that

$$
\sup _{0 \leqslant r<1} \int_{0}^{2 \pi} \log ^{+}\left|F\left(r e^{i \theta}\right)\right| d \theta<\infty .
$$

Functions of this class have non-tangential limits at almost all points of $\{z:|z|=1\}$ ([34]).

THEOREM 1.2.1. The general function $F$, of class $N$ can be represented as:

$$
F(z)=B(z) \exp \left\{\frac{1}{2 \pi} \int_{0}^{2 \pi} \frac{e^{i t}+z}{e^{i t}-z} d \lambda(t)\right\}
$$

where $B(z)$ is a Blaschke product, and $\lambda(\cdot)$ is a real-valued function of bounded variation ([34]).

Let $N^{1}$ be the subclass of $N$ made up of functions $F$, such that the function $\lambda$ corresponding to $F$ in (1.2.2) has its posititve variation absolutely continuous.

THEOREM 1.2.2. A function $F \in N$, is in $N^{1}$ if and only if,

$$
\lim _{r \rightarrow 1} \int_{0}^{2 \pi} \log ^{+}\left|F\left(r e^{i \theta}\right)\right| d \theta=\int_{0}^{2 \pi} \log ^{+}\left|F\left(e^{i \theta}\right)\right| d \theta .
$$

THEOREM 1.2.3. If $\varphi(u)$ is non-decreasing and convex in $(-\infty, \infty)$ and $F$ is analytio in $\{z:|z| \leqslant 1\}$, then $\int_{0}^{2 \pi} \varphi\left(\log \left|F^{\prime}\left(r e^{i 0}\right)\right|\right) d \theta$ is a non-decreasing function of $r$, for $0 \leqslant r<1$.

THeorem 1.2.4. If $\varphi$ is as in Theorem 1.2.3 and $\mathbb{F} \in N^{1}$, then

$$
\int_{0}^{2 \pi} \varphi\left(\log ^{+}\left|F^{\prime}\left(r e^{i \theta}\right)\right|\right) d \theta \leqslant \int_{0}^{2 \pi} \varphi\left(\log ^{+}\left|F\left(e^{i \theta}\right)\right|\right) d \theta .
$$

The preceeding three theorems are from ([34]).
LEMMa 1.2.1. Let $f$ be continuous on $[0,2 \pi]$ and define;

$$
F\left(r e^{i \theta}\right)=P f\left(r e^{i \theta}\right)=\frac{1}{2 \pi} \int_{0}^{2 \pi} P_{r}(\theta-t) f(t) d t
$$

where $P_{r}(\theta-t)$ is the Poisson kernel. Then $F \in N^{1}$.

Proof. Recall that $P_{r}(\cdot)$ is defined as,

$$
P_{r}(\theta)=\operatorname{Re}\left(\frac{e^{i t}+r e^{i \theta}}{e^{i t}-r e^{i \theta}}\right)=\frac{1-r^{2}}{1-2 r \cos (\theta)+r^{2}} .
$$

Since $f$ is continuous on $[0,2 \pi]$, it is bounded there. So let $|f(t)| \leqslant m$ for all $t \in[0,2 \pi]$. In addition $F^{\prime}\left(r e^{i \theta}\right) \rightarrow f(\theta)$ uniformly in $\theta, F$ is analytic on $\{z:|z|<1\}$, and $F$ is continuous on $\{z:|z| \leqslant 1\}$. (See [13]). It follows from the Maximum Principle that $\left|F\left(r e^{i \theta}\right)\right| \leqslant m$ for all $0 \leqslant r<1$ and all $\theta$. Hence,

$$
\log ^{+}\left|F\left(r e^{i \theta}\right)\right| \leqslant \log ^{+} m \quad \text { for ail } r \in[0,1) \text { and all } \theta .
$$

It follows that,

$$
\int_{0}^{2 \pi} \log ^{+}\left|F\left(r e^{i \theta}\right)\right| d \theta \leqslant \int_{0}^{2 \pi} \log ^{+} m=2 \pi \log ^{+} m \quad \text { for all } r .
$$

But then $F \in N$ by definition. By Theorem 1.2.2, $F^{\prime}$ will be in $N^{1}$ if (1.2.3) holds. But by the Lebesgue Dominated Convergence Theorem and above,

$$
\lim _{r \rightarrow 1} \int_{0}^{2 \pi} \log ^{+}\left|F^{\prime}\left(r e^{i \theta}\right)\right| d \theta=\int_{0}^{2 \pi} \lim _{r \rightarrow 1} \log ^{+}\left|F^{\prime}\left(r e^{i \theta}\right)\right| d \theta=\int_{0}^{2 \pi} \log ^{+}\left|F^{\prime}\left(e^{i \theta}\right)\right| d \theta
$$

since $\log ^{+}(\cdot)$ is continuous. Hence $F \in N^{1}$.

Let

$$
\mu_{\varphi}(r ; F)=\int_{0}^{2 \pi} \varphi\left(\left|F\left(r e^{i \theta}\right)\right|\right) d \theta \quad \text { and } \quad \mu_{\varphi}(F)=\sup _{0 \leqslant r<1} \mu_{\varphi}(r ; F) .
$$

Note that since $\varphi$ is log-convex, $\mu_{\varphi}\left(r ; F^{\prime}\right)$ is non-decreasing in $r$ by Theorem 1.2.3. Hence

$$
\mu_{\varphi}(F)=\lim _{r \rightarrow 1} \mu_{\varphi}\left(r ; F^{\prime}\right) .
$$

Now define;

(1.2.5) $\quad H^{* \varphi}=\left\{F: F\right.$ is analytic on $\{z:|z|<1\}$ and $\left.\mu_{\varphi}(F)<\infty\right\}$ and;

(1.2.6) $\quad H^{\varphi}=\left\{F: F\right.$ is analytic on $\{z:|z|<1\}$ and $\mu_{\varphi}(\lambda F)<\infty$ for some $\lambda>0\}$. 
DEFINITION 1.2.2. $H^{*}$ and $H^{\varphi}$ are called the Hardy-Orlicz class of functions.

Propostrion 1.2.1. A necessary and sufficient condition for $H^{*}=H^{q}$ is that $\varphi \in \Delta_{2}$.

In the following $L^{\varphi}=L^{\varphi}(0,2 \pi)$.

THeorem 1.2.5. Let $F \in N^{1}$ and $F\left(e^{i}\right) \in L^{*}$. Then $F \in H^{*} \varphi$.

CoRoLLARY 1.2.1. Let $\varphi \in \Delta_{2}$ and $F \in N^{1}$. Then $F \in H^{\varphi}$ if $F\left(e^{i}\right) \in L^{\varphi}$. An $F$-norm can be defined on $H^{\varphi}$ by

$$
\left\|W^{\prime}\right\|_{H_{\varphi}}=\sup _{0 \leqslant r<1}\left\|F^{\prime}\left(r e^{i \cdot}\right)\right\|_{\varphi}
$$

where the norm on the right is the $F$-norm generated by $\varphi$ on $L^{\varphi}$. The classes $H^{\varphi}$ with the $H^{\prime}$-norm $\|\cdot\|_{H_{\infty}}$ are Fréchet spaces, and are called the Hardy-Orlicz spaces. These spaces were defined for a convex $p$ by Weiss in [31].

LeMara 1.2.2. If $F \in H_{\varphi}$ then $F\left(e^{i}\right) \in L_{\varphi}(0,2 \pi)$.

LEMMA 1.2.3. If $F \in H_{\varphi}$, then $\mu_{\varphi}(F)=\int_{0}^{2 \pi} \varphi\left(\left|F\left(e^{i \theta}\right)\right|\right) d \theta$.

THEOREM 1.2.6. If $F \epsilon H_{\varphi}$, then $\left\|F^{\prime}\right\|_{I_{\varphi}}=\left\|F\left(e^{i}\right)\right\|_{\varphi}$.

LEMMA 1.2.4. If $p \in \Delta_{2}$ and $F \in H_{p}$, then there exists $\left\{F_{n}\right\} \subset H_{p}$ such that $F_{n}$ is continuous in $\{z:|z| \leqslant 1\}$ and $\lim _{n \rightarrow \infty}\left\|F-F_{n}\right\|_{I I_{\varphi}}=0$.

LEMTA 1.2.5. If $\varphi \in \Delta_{2}$ and $F \in H_{\varphi}$, then

$$
\lim _{R \rightarrow 1}\left\|H^{\prime}(\cdot)-F^{\prime}(R \cdot)\right\|_{H_{\varphi}}=0 .
$$

THEOREM 1.2.7. Polynomials are dense in $H_{\varphi}$ if $\varphi \in \Delta_{2}$.

In the case $\varphi(u)=\psi\left(u^{s}\right)$ where $0<s \leqslant 1$ and $\psi$ is a $\varphi$-function, an $s$-homogeneous norm can be defined in $H_{p}$ by means of the $s$-homogeneous norm in $L^{\varphi}$ as;

$$
\|F\|_{s H_{q}}=\sup _{0 \leqslant r<1}\left\|F\left(r e^{i}\right)\right\|_{s p} .
$$

The $F$-norm $\|\cdot\|_{B \dot{H_{\varphi}}}$ is equivalent to the norm $\|\cdot\|_{H_{\varphi}}$ and. Theorems 1.2.6 and 1.2.7 hold using $\|\cdot\|_{s H_{\varphi}}$ and $\|\cdot\|_{s_{\varphi}}$ instead of $\|\cdot\|_{H_{\varphi}}$ and $\|\cdot\|_{\varphi}$.

1.3. Results concerning subharmonic functions. In this section the three line theorem for subharmonic functions, and certain related results will be given.

THEOREM 1.3.1. (THE TEREE LINE LEMCMA FOR SUBHARMONIO FUNOTIONS). Let $f(z)$ be non-negative, bounded and defined in $S=\{z: 0$ $\leqslant \operatorname{Re} z \leqslant 1\}$ such that $\log f(z)$ is subharmonic in $\{z: 0<\operatorname{Re} z<1\}$ and continuous in $S$. If $f(0+i y) \leqslant M_{1}$, and $f(1+i y) \leqslant M_{2}$, then $f(t+i y)$ $\leqslant M_{1}^{1-t} M_{2}^{t}$.
LEMMA 1.3.1. If $f(z)$ is non-negative and $\log f(z)$ is subharmonic in a domain $D$, then $(f(z))^{a}$ is subharmónic in $D$ for all $a>0$.

Proof. Let $z \in D$ and let $\{\xi:|z-\xi| \leqslant \varrho\} \subset D$. Then by definition:

$$
\log f(z) \leqslant \frac{1}{2 \pi} \int_{0}^{2 \pi} \log f\left(z+\varrho e^{i \theta}\right) d \theta
$$

Therefore;

$$
\begin{aligned}
(f(z))^{a} & =e^{\alpha \log f(z)} \leqslant e^{\alpha \frac{1}{2 \pi} \int_{0}^{2 \pi} \log f\left(z+\rho e^{i \theta}\right) d \theta} \\
& =e^{\frac{1}{2 \pi} \int_{0}^{2 \pi} \log \left(f\left(z+e e^{i \theta}\right)\right)^{a} d \theta} \leqslant \frac{1}{2 \pi} \int_{0}^{2 \pi}\left(f\left(z+\varrho e^{i \theta}\right)\right)^{a} d \theta,
\end{aligned}
$$

by Jensen's inequality. Hence $(f(z))^{\alpha}$ is subharmonic.

DEFINITION 1.3.1. A function $I(\cdot)$ defined and continuous in the strip $0 \leqslant \operatorname{Re}(z) \leqslant 1$ will be called of admissible growth if

$$
\sup _{|y| \leqslant r} \sup _{0 \leqslant x \leqslant 1} \log |I(x+i y)| \leqslant A e^{\alpha r}, \quad . a<\pi .
$$

The following result is stated by Hirschman [11] for analytic funetions, but an examination of the proof shows it proves actually the following result.

LEMMA 1.3.2. (Hirschman [11]): Let $I(z)$ be non-negative and $\log I(z)$ be subharmonic and continuous in $0 \leqslant \operatorname{Re} z \leqslant 1$. If $I(z)$ is of admissible growth, and

$$
\log I(i y) \leqslant \alpha_{0}(y) \quad \text { and } \quad \log I(1+i y) \leqslant \alpha_{1}(y),
$$

then for all $t \in[0,1]$,

where

$$
\log (I(t)) \leqslant \int_{-\infty}^{\infty} \omega(1-t, y) \alpha^{0}(y) d y+\int_{-\infty}^{\infty} \omega(t, y) \alpha_{1}(y) d y
$$

$$
\omega(t, y)=\frac{\frac{1}{2} \tan \left(\frac{\pi t}{2}\right)}{\left[\tan ^{2}\left(\frac{\pi t}{2}\right)+\tanh ^{2}\left(\frac{\pi y}{2}\right)\right] \cosh ^{2}\left(\frac{\pi y}{2}\right)} .
$$

\section{INTERPOLATION OF SUBLINEAR OPERATORS}

In this section, the M. Riesz convexity theorem [27] is generalized to sublinear operators on $L^{\varphi}$ classes of Banach space valued functions and to $\mathbb{H}^{\varphi}$ classes of functions on the disc. 
2.1. Interpolation in generalized Orlicz spaces. It is convenient to introduce the following:

DEFINITION 2.1.1. Let $(\Omega, \Sigma, \mu)$ be a measure space, and $X$ a Banach space. Let $\varphi$ be a $\varphi$-function and

$$
\mathscr{F}=\{f: f: \Omega \rightarrow X \text { and } f \text { is strongly measurable }\} .
$$

Define $\|f\|_{\varphi(X)}=\|\| f\left\|_{X}\right\|_{\varphi}$ for $f \epsilon \mathscr{F}$, and $L^{\varphi(X)}=\left\{f: f \epsilon \mathscr{F}\right.$ and $\left.\|f\|_{\varphi(X)}<\infty\right\}$. Then $L^{\varphi(X)}$ is called the Orlicz space of $X$-valued functions.

All the properties of $L^{p}$ discussed in Section. 1 hold for $L^{p(X)}$.

Definition 2.1:2. Let $\left(\Omega_{1}, \Sigma_{1}, \mu\right)$ and $\left(\Omega_{2}, \Sigma_{2}, v\right)$ be measure spaces and $X, Y$ be Banach spaces. Let $\mathscr{F}=\left\{f: f: \Omega_{1} \rightarrow Y\right.$ and $f$ is strongly measurable $\}$ and $\mathscr{G}=\left\{f: f: \Omega_{2} \rightarrow X, f\right.$ strongly measurable $\}$. Suppose $T$ is a mapping of a subclass of $\mathscr{F}$ into $\mathscr{G}$. Then $T$ is called a sublinear operator if it satisfies the following properties:

(i) If $f=f_{1}+f_{2}$ and $T f_{i}(i=1,2)$ are defined, then $I f$ is defined.

(ii) $\left\|T\left(f_{1}+f_{2}\right)\right\|_{X} \leqslant\left\|T f_{1}\right\|_{X}+\left\|\dot{T} f_{2}\right\|_{X}$.

(iii) For any scalar $a,\|T(a f)\|_{X}=|a|\|T f\|_{X}$.

Remark. If $\varphi(u)=\psi\left(u^{s}\right)$ for $0<s \leqslant 1$ and $\psi$ a convex $p$-function, then $\|f\|_{s \varphi}=\left\||f|^{s}\right\|_{1 p}, f \in L^{\varphi}$. For:

$$
\begin{aligned}
\|f\|_{s \varphi} & =\inf \left\{\varepsilon: \int_{\Omega} \varphi\left(\frac{|f|}{\varepsilon^{1 / s}}\right) d \mu \leqslant 1\right\} \\
& =\inf \left\{\varepsilon: \int_{\Omega} \psi\left(\frac{|f|^{s}}{\varepsilon}\right) d \mu \leqslant 1\right\}=\left\||f|^{s}\right\|_{1 \psi} .
\end{aligned}
$$

Similarly $\|f\|_{s p(X)}=\|\| f\left\|_{X}^{s}\right\|_{1 w}$.

The main result of this section is given in the following:

THEOREM 2.1.1. Let $\varphi_{i}, Q_{i}(i=1,2)$ be p-functions such that $\varphi_{i}(u)$ $=\tilde{\psi_{i}}\left(u_{i}^{r_{i}}\right) ; Q_{i}(u)=S_{i}^{\tilde{N}}\left(u^{s_{i}}\right)$ where $0<r_{i}, s_{i} \leqslant 1$ and $\psi_{i}^{\tilde{n}}, S_{i}^{\tilde{n}}$ are convex. Let $\mathscr{F}$ and $\mathscr{G}$ be as in Definition 2.1.2, and let $L^{p_{i}(Y)}$ be Orlicz spaces on $\left(\Omega_{1}, \Sigma_{1}, \mu\right)$ and $L^{Q_{i}(X)}$ be Orlicz spaces on $\left(\Omega_{2}, \Sigma_{2}, v\right)$. Suppose $T$ is a sublinear mapping $L^{\varphi_{i}(Y)}$ into $L^{Q_{i}(X)}$ satisfying;

$$
\begin{array}{ll}
\|T f\|_{r Q_{1}(X)} \leqslant M_{1}\|f\|_{r \varphi_{1}(X)} & \text { for all } f \in L^{p_{1}(Y)}, \\
\|T f\|_{r Q_{2}(X)} \leqslant M_{2}\|f\|_{r \varphi_{2}(X)} & \text { for all } f \in L^{p_{2}(X)}
\end{array}
$$

for some $r \leqslant \min \left\{s_{1}, s_{2}, r_{1}, r_{2}\right\}$. If $\mathscr{L}(Y)$ is the olass of simple functions on $\mathscr{F}$, then

$$
\text { : }\|T f\|_{r_{Q_{t}(X)}} \leqslant 4 M_{1}^{1-t} M_{2}^{t}\|f\|_{r \varphi_{t}(Y)} \quad \text { for all } f \in \mathscr{L}(X)
$$

where $Q_{t}^{-1}=\left(Q_{1}^{-1}\right)^{-1-t}\left(Q_{2}^{-1}\right)^{t}$ and $\varphi_{t}^{-1}=\left(\varphi_{1}^{-1}\right)^{1-t}\left(\varphi_{2}^{-1}\right)^{t}$ with $0 \leqslant t \leqslant 1$.
Proof. Let $\psi_{i}(u)=\tilde{\psi_{i}}\left(u^{r_{i} / r}\right)$ and $S_{i}(u)=S_{i}^{\tilde{i}}\left(u^{s_{i} / r}\right)$. Since $r \leqslant r_{i}, s_{i} ; \psi_{i}$ and $S_{i}$ are convex, and $\varphi_{i}(u)=\psi_{i}\left(u^{r}\right), Q_{i}(u)=S_{i}\left(u^{r}\right)$. So the norms in (2.1.1) make sense. Let $\psi_{t}^{-1}(u)=\left(\psi_{1}^{-1}(u)\right)^{1-t}\left(\psi_{2}^{-1}(u)\right)^{t}$ and $S_{t}^{-1}(u)$ $=\left(S_{1}^{-1}(u)\right)^{1-t}\left(S_{2}^{-1}(u)\right)^{t}$. Then if follows that $\varphi_{t}(u)=\psi_{t}\left(u^{r}\right), \psi_{t}$ convex and $Q_{t}(u)=S_{t}\left(u^{r}\right), S_{t}$ convex.

Let $R_{1}, R_{2}$ be the complementary functions to $S_{1}, S_{2}$ and $R_{t}(u)$ $=\left(R_{1}^{-1}(u)\right)^{1-t}\left(R_{2}^{-1}(u)\right)^{t}$.

Let $\alpha_{i}(u)=\varphi_{i}^{-1}(u), \alpha_{t}(u)=\alpha_{1}^{1-t}(u) \alpha_{2}^{t}(u)$, and define $\alpha_{z}(u)=\alpha_{1}^{1-z}(u) \times$ $\times a_{2}^{z}(u)$, where $z=x+i y$ is a complex number. Then for each $u \neq 0$, since $\alpha_{i}(\cdot)$ is positive, $\alpha_{z}(u)$ is an analytic function of $z$ in the strip $0 \leqslant x \leqslant 1$. Similarly let $\beta_{i}=R_{i}^{-1}(u)$ and then $\beta_{z}(u)=\beta_{1}^{1-z}(u) \beta_{2}^{z}(u)$ is also analytic in $0 \leqslant x \leqslant 1$. It follows that

$$
\begin{aligned}
\left|\alpha_{z}(u)\right| & =\left|\alpha_{1}(u)\right|^{1-z}\left|\alpha_{2}(u)\right|^{z}=\alpha_{1}(u)^{1-x} \alpha_{2}(u)^{x} \\
& \leqslant \max _{x}\left\{\alpha_{1}^{1-x}(u) \alpha_{2}^{x}(u)\right\} \leqslant \max \left\{1, \alpha_{1}(u)\right\} \cdot \max \left\{1, \alpha_{2}(u)\right\}
\end{aligned}
$$

i.e. $\alpha_{z}(u)$ is bounded in $0 \leqslant x \leqslant 1$ for each $u$. Similarly $\beta_{z}(u)$ is bounded in $0 \leqslant x \leqslant 1$ for each $u$.

Let $f \in \mathscr{L}(Y) \subset L^{\varphi_{i}(Y)}$. Then $T f$ is well defined, so consider,

$$
\|\| T f\left\|_{X}^{r}\right\|_{S_{t}}=\sup \left\{\int_{\Omega_{2}}\|T f\|_{X}^{r}|g| d v:\|g\|_{1 R_{t}} \leqslant 1, g \mathscr{L}_{v}\right\}
$$

where $\mathscr{L}_{v}$ is the set of simple functions on $\left(\Omega_{2}, \Sigma_{2}, v\right)$. The norm defined by (2.1.3) is equivalent to the Orlicz norm, $\|\cdot\|_{S_{t}}^{i}=\sup \left\{\int_{\Omega} f g d \mu:\|g\|_{1} S_{t}^{\sim}\right.$ $\leqslant 1, g \in \mathscr{L}(\Omega, \Sigma, \mu)\}$ where $S_{t}^{\tilde{t}}$ is the complementary function to $S_{t}$ (see [24]).

Suppose that $\|f\|_{r \varphi_{t}}=1$, and fix $g \in \mathscr{L}_{v}$ such that $\|g\|_{1 R_{t}} \leqslant 1$ and consider

But $g \in \mathscr{L}_{\text {p implies }}$

$$
I=\int_{\Omega_{2}}\|T f\|_{X}^{r}|g| d \nu
$$

Now define,

$$
g=\sum_{l=1}^{m_{2}} b_{l} \chi_{G_{l}}=\sum_{l=1}^{m_{2}}\left|b_{l}\right| e^{i \theta_{l}} \chi_{G_{l}} .
$$

$$
G_{z}=\beta_{z}\left(R_{t}|g|\right) e^{i \theta}=\sum_{l=1}^{m_{2}} \beta_{z}\left(R_{t}\left|b_{l}\right|\right) e^{i \theta} \chi_{\theta_{l}} .
$$

Since $f \epsilon \mathscr{L}(\bar{Y}), f=\sum_{j=1}^{m_{1}} \alpha_{j} \chi_{F_{j}}, \alpha_{j} \in Y$. Write $\alpha_{j}=a_{j} u_{j}$ where $a_{j}=\left\|\alpha_{j}\right\|_{Y}$ and $\left\|u_{j}\right\|_{Y}=1$. Then $f=\sum_{j=1}^{m_{1}} a_{j}\left(u_{j} \chi_{F_{j}}\right)$ and define

$$
F_{z}=\sum_{j=1}^{m_{1}} \alpha_{z}\left(\varphi_{t}\left(a_{j}\right)\right)\left(u_{j} \chi_{F_{j}}\right) .
$$


Since the $F_{j}$ 's are disjoint,

so that

$$
\|f\|_{Y}=\sum_{j=1}^{m_{1}} a_{j}\left\|u_{j}\right\|_{Y} \chi_{F_{j}}=\sum_{j=1}^{n_{1}} a_{j} \chi_{F_{j}} .
$$

$$
\left\|F_{z}\right\|_{Y}=\sum_{j=1}^{m_{1}}\left|\alpha_{z}\left(\varphi_{t}\left(a_{j}\right)\right)\right| \chi_{F_{j}}=\left|\alpha_{z}\left(\varphi_{t}\left(\|f\|_{Y}\right)\right)\right| .
$$

Since $F_{z} \in \mathscr{L}(Y)$ and $G_{z} \in \mathscr{L}_{\nu}$ the following extension of (2.1.4) can be defined,

$$
I(z)=\int_{\Omega_{2}}\left\|T\left(F_{z}\right)\right\|_{x}^{r}\left|G_{z}\right| d \nu .
$$

It is clear by construction that $I(t)=I$. The plan of the proof is to show that $I(z)$ satisfies the hypothesis of the three line theorem for subharmonic functions (Theorem 1.3.1) and obtain (2.1.2) as a consequence of that theorem. $I(z)$ can be simplified using (2.1.5) and (2.1.6) to yield;

$$
I(z)=\sum_{l=1}^{m_{2}} \int_{G_{l}}\left\|T\left(\beta_{z}^{1 / r}\left(R_{t}\left(\left|b_{l}\right|\right)\right) F_{z}\right)\right\|_{x}^{r} d \nu
$$

where the defining property (iii) for sublinear operators is used here. To simplify things, let

$$
\left\{\begin{array}{l}
\gamma_{z}^{l}=\sum_{j=1}^{m_{1}} \beta_{z}^{1 / r}\left(R_{t}\left(\left|b_{l}\right|\right)\right) \alpha_{z}\left(\phi_{t}\left(a_{j}\right)\right) u_{j} \chi_{F_{j}}, \\
\lambda_{j}^{l}(z)=\sum_{j=1}^{m_{1}} \beta_{z}^{1 / r}\left(R_{t}\left(\left|b_{l}\right|\right)\right) \alpha_{z}\left(\varphi_{t}\left(a_{j}\right)\right) \\
\Gamma_{l}(z)=\int_{\sigma_{l}}\left\|T\left(\gamma_{z}^{l}\right)\right\|_{X}^{r} d v
\end{array}\right.
$$

Then $\gamma_{z}^{l}=\sum_{j=1}^{m_{1}} \lambda_{j}^{l}(z) u_{j} \chi_{F_{j}}$ and $I(z)=\sum_{l=1}^{m_{2}} \Gamma_{l}(z)$. It is clear that $\lambda_{j}^{l}(z)$ is analytic, bounded, and continuous in $0 \leqslant x \leqslant 1$. From now on the proof proceeds in steps.

Step 1. $T_{l}(z)$ is continuous in $0 \leqslant x \leqslant 1$. For, consider

$$
\begin{gathered}
\left|\Gamma_{l}(z+\Delta z)-\Gamma_{l}(z)\right| \leqslant \int_{\sigma_{l}}\left|\left\|T\left(\gamma_{z+\Delta z}^{l}\right)\right\|_{X}^{r}-\left\|T\left(\gamma_{z}^{l}\right)\right\|_{X}^{r}\right| d v \\
\leqslant \int_{\sigma_{l}}\left|\left(\left\|T\left(\gamma_{z+\Delta z}^{l}\right)\right\|_{X}-\left\|T\left(\gamma_{z}^{l}\right)\right\|_{X}\right)^{r}\right| d \nu \\
\quad \quad\left(\text { since } u^{r}, 0<r \leqslant 1,\right. \text { is subadditive) } \\
\leqslant \int_{\sigma_{l}}\left\|T\left(\gamma_{z+\Delta z}^{l}-\gamma_{z}^{l}\right)\right\|_{X}^{r} d \nu
\end{gathered}
$$

(since $T$ is sublinear),

$$
\leqslant 2\|\| T\left(\gamma_{z+\Delta z}^{l}-\gamma_{z}^{l}\right)\left\|_{X}^{r}\right\|_{L S_{1}}\left\|\chi_{G_{l}}\right\|_{1 R_{1}}
$$

(by Hölder's inequality),

$$
\leqslant 2\left\|T\left(\gamma_{z+\Delta z}^{l}-\gamma_{z}^{l}\right)\right\|_{r Q_{1}(X)}\left\|\chi_{G_{l}}\right\|_{1 R_{1}}
$$

(by the Remark above the statement of the Theorem)

$$
\leqslant 2 M_{1}\left\|\gamma_{z+\Delta z}^{l}-\gamma_{z}^{l}\right\|_{r q_{1}(Y)}\left\|\chi_{G_{l}}\right\|_{1 R_{1}},
$$

by hypothesis, since $\gamma_{z}^{l} \in \mathscr{L}(Y) \subset L^{q_{1}(Y)}$. But

Therefore

$$
\left\|\gamma_{z+\Delta z}^{l}-\gamma_{z}^{l}\right\|_{r_{1}(Y)} \leqslant \sum_{j=1}^{m_{1}}\left|\lambda_{j}^{l}(z+\Delta z)-\lambda_{j}^{l}(z)\right|^{r}\left\|u_{j} \chi_{F_{j}}\right\|_{r q_{1}(Y)} .
$$

$$
\lim _{\Delta z \rightarrow 0}\left\|\gamma_{z+\Delta z}^{l}-\gamma_{z}^{l}\right\|^{r \varphi_{1}(Y)} \leqslant \sum_{j=1}^{m_{1}} \lim _{\Delta z \rightarrow 0}\left|\lambda_{j}^{l}(z+\Delta z)-\lambda_{j}^{l}(z)\right|^{r}\left\|u_{j} \chi_{F_{j}}\right\|_{r q_{1}(Y)}=0
$$

since $\lambda_{j}^{l}(z)$ is continuous and the sum is finite. It follows from (2.1.10) that

$$
\lim _{\Delta z \rightarrow 0}\left|\Gamma_{l}(z+\Delta z)-\Gamma_{l}(z)\right|=0
$$

and therefore $\Gamma_{l}(z)$ is continuous in $0 \leqslant x \leqslant 1$.

Step 2. $\Gamma_{l}(z)$ is bounded in $0 \leqslant x \leqslant 1$. For, as in (2.1.10),

$$
\begin{aligned}
\Gamma_{l}(z) & =\int_{G_{l}}\left\|T \gamma_{z}^{l}\right\|_{X}^{r} r^{2} d v \leqslant 2\left\|T \gamma_{z}\right\|_{r Q_{1}(X)}\left\|\chi_{G_{l}}\right\|_{1 R_{1}} \\
& \leqslant 2 M_{1}\left\|\gamma_{z}\right\|_{r \varphi_{1}(Y)}\left\|\chi_{G_{l}}\right\|_{1 R_{1}} \\
& \leqslant 2 M_{1} \sum_{j=1}^{m_{1}} \mid \lambda_{j}^{l}(z) r^{r}\left\|u_{j} \chi_{F_{j}}\right\|_{r \Phi_{1}(Y)}\left\|\chi_{G_{l}}\right\|_{1 R_{1}} .
\end{aligned}
$$

It follows that $\Gamma_{l}(z)$ is bounded in $0 \leqslant x \leqslant 1$ since $\lambda_{j}^{l}(z)$ is bounded in $0 \leqslant x \leqslant 1$ and the sum is finite.

Step 3. $\log \Gamma_{l}(z)$ is subharmonic in $0<x<1$. For, let $h(z)$ be any harmonic function in $0<x<1$, and let $H(z)$ be the analytic function whose real part is $h(z)$. If $e^{h(z)} \Gamma_{l}(z)$ is subharmonic for all such $h(z)$, then $\log \Gamma_{l}(z)$ will be subharmonic. Since a function is subharmonic in a region if it is subharmonic in a neighborhood of each point, fix $z \in\{0<x<1\}$, take $\varrho>0$, and let $z_{1}, z_{2}, \ldots, z_{p}$ be a set of points equally spaced on the circle of radius $\varrho$ about $z$. Then it is sufficient to show

$$
e^{\dot{h}(z)} \Gamma_{l}(z) \leqslant \frac{1}{2 \pi} \int_{0}^{2 \pi} e^{h\left(z+e e^{i \theta}\right)} \Gamma_{l}\left(z+\varrho e^{i \theta}\right) d \theta .
$$

Let $\gamma_{z}^{* l}=e^{\frac{1}{r} H(z)} \gamma_{z}^{l}$ and $\lambda_{j}^{* l}(z)=e^{\frac{1}{r} H(z)} \lambda_{j}^{l}(z)$. Then $\gamma_{z}^{* l}=\sum_{j=1}^{m_{1}} \lambda_{j}^{* l}(z)\left(u_{j} \chi_{F_{j}}\right)$, 
and

$$
I_{l}^{*}(z)=e^{h(z)} \Gamma_{l}(z)=\int_{G_{l}}\left\|T \gamma_{z}^{*}\right\|_{X}^{r} d v
$$

Now it will be sufficient to show that $\log \left\|T \gamma_{z}^{*{ }^{*}}\right\|_{X}$ is subharmonic for each $\dot{\omega}$, for if the latter holds, then by Lemma 1.3.1, $\left\|T\left(\gamma_{z}^{* z}\right)\right\|_{X}^{r}$ would be subharmonic for each $\omega$.

In this case, then

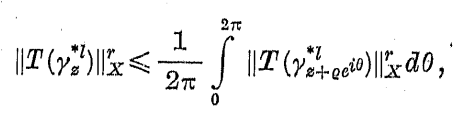

for each $\omega$, and then

$$
\begin{aligned}
\Gamma_{l}^{*}(z) & =\int_{\sigma_{l}}\left\|T\left(\gamma_{z}^{* l}\right)\right\|_{X}^{*} d \nu \leqslant \int_{\sigma_{l}}\left(\frac{1}{2 \pi} \int_{0}^{2 \pi}\left\|T\left(\gamma_{z+e e^{i \theta}}^{* l}\right)\right\|_{X}^{*} d l\right) d \nu \\
& =\frac{1}{2 \pi} \int_{0}^{2 \pi} \int_{\sigma_{l}} \| T\left(\gamma_{\left.z+e e^{i \theta}\right)} \|_{X}^{r} d \nu d \theta=\frac{1}{2 \pi} \int_{0}^{2 \pi} \Gamma_{l}^{*}\left(z+\varrho e^{i 0}\right) d \theta .\right.
\end{aligned}
$$

It then follows that $\Gamma_{l}^{*}(z)$ is subharmonic and so $\log \Gamma_{l}(z)$ would be subharmonic.

To show $\log \left\|T\left(\gamma_{2}^{* l}\right)\right\|_{X}$ is subharmonic, it is sufficient to show $e^{k_{(z)}(z)}\left\|T_{\gamma_{z}}^{* l}\right\|_{X}$ is subharmonic where $k(z)$ is any harmonic function. Let $K(z)$ be the

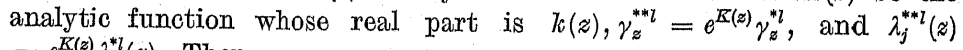
$=e^{K(z)} \lambda_{j}^{* l}(z)$. Then

and

$$
\gamma_{z}^{* * l}=\sum_{j=1}^{m_{1}} \lambda_{j}^{* * l}(z) u_{j} \chi_{F_{j}}
$$

$$
e^{k(z)}\left\|T \gamma_{z}^{*}\right\|_{X}=\left|e^{K(z)}\right|\left\|T \gamma_{z}^{*}\right\|_{X}=\left\|T_{\gamma^{*}}^{* *}\right\|_{X}
$$

Since $K(z), H(z)$ and $\lambda_{j}^{l}(z)$ are analytic, it follows that $\lambda_{j}^{* * k}(z)$ is analytic and therefore

$$
\lambda_{j}^{* * l}(z)=\frac{1}{2 \pi} \int_{0}^{2 \pi} \lambda_{j}^{* * l}\left(z+\varrho e^{i \theta}\right) d \theta=\lim _{p \rightarrow \infty} \frac{1}{p} \sum_{n=1}^{p} \lambda_{j}^{* * t}\left(z_{n}\right)
$$

where $z_{n}=\varrho e^{i \Delta \theta_{n}}$ and $\Delta \theta_{n}=\frac{2 \pi}{p}$. Consider

(2.1.16) $\left\|T \gamma_{z}^{* *}\right\|_{X} \leqslant\left|\left\|T \gamma_{z}^{* * \tau}\right\|_{X}-\left\|T\left(\frac{1}{p} \sum_{n=1}^{p} \gamma_{z_{n}}^{* * \tau}\right)\right\|_{X}\right|+\left\|T\left(\frac{1}{p} \sum_{n=1}^{p} \gamma_{z_{n}}^{* * l}\right)\right\|_{X}$.
But

$$
\begin{aligned}
\left\|T \gamma_{z}^{* *}\right\|_{X}-\| T\left(\frac{1}{p} \sum_{n=1}^{p} \gamma_{z_{n}}^{* *}\right) & \left\|_{X} \mid \leqslant\right\| T\left(\gamma_{z}^{* * l}-\frac{1}{p} \sum_{n=1}^{p} \gamma_{z_{n}}^{* * l}\right) \|_{X} \\
& \leqslant \sum_{j=1}^{m_{1}}\left|\lambda_{j}^{* * l}(z)-\frac{1}{p} \sum_{n=1}^{p} \lambda_{j}^{* * l}\left(z_{n}\right)\right|\left\|u_{j} \chi_{F_{j}}\right\|_{X} .
\end{aligned}
$$

Therefore, for each $\omega$

$$
\begin{aligned}
& \lim _{p \rightarrow \infty}\left|\left\|T \gamma_{z}^{* * \tau}\right\|_{X}-\left\|T\left(\frac{1}{p} \sum_{n=1}^{p} \gamma_{z_{n}}^{* * \tau}\right)\right\|_{X}\right| \\
& \leqslant \sum_{j=1}^{m_{1}} \lim _{p \rightarrow \infty}\left|\lambda_{j}^{* * \tau}(z)-\frac{1}{p} \sum_{n=1}^{p} \lambda_{j}^{* * \tau}\left(z_{n}\right)\right|\left\|u_{j} \chi_{F_{j}}\right\|_{X}=0, \quad \text { by (2.1.15) }
\end{aligned}
$$

It follows from (2.1.16) that

$$
\left\|T \gamma_{z}^{* * t}\right\|_{X} \leqslant \lim _{p \rightarrow \infty} \frac{1}{p} \sum_{n=1}^{p}\left\|T \gamma_{z_{n}}^{* * t}\right\|_{X}=\frac{1}{2 \pi} \int_{0}^{2 \pi} \| T \gamma_{z+e e^{i \theta} \|_{X}}^{* * l} d \theta
$$

for each $\omega$. This is the same as

$$
e^{k(z)}\left\|T \gamma_{z}^{* l}\right\|_{X} \leqslant \frac{1}{2 \pi} \int_{0}^{2 \pi} e^{k\left(z+\rho e^{i \theta}\right)}\left\|T \gamma_{z+\rho e^{i \theta}}^{* l}\right\|_{X} d \theta
$$

which implies $\log \left\|T \gamma_{z}^{* z}\right\|_{x}$ is subharmonic for each $\omega$. Hence, by the previous argument $\log \Gamma_{l}(z)$ is subharmonic in $0<x<1$.

It thus follows that, since $I(z)=\sum_{l=1}^{m_{2}} \Gamma_{l}(z), I(z)$ is bounded and continuous in $0 \leqslant x \leqslant 1$.

Step 4. $I(i y) \leqslant 2 M_{1}$ and $I(1+i y) \leqslant 2 M_{2}$. For, consider,

$$
\begin{aligned}
I(i y) & =\int_{\Omega}\left\|T F_{i y}\right\|_{X}^{r}\left|G_{i y}\right| d \nu \leqslant 2\|\| T F_{i y}\left\|_{X}^{r}\right\|_{1 S_{t}}\left\|G_{i y}\right\|_{1 R_{t}} \\
& =2\left\|T F_{i y}\right\|_{r Q_{t}(X)}\left\|G_{i y}\right\|_{1 R_{t}} \leqslant 2 M_{1}\left\|F_{i y}\right\|_{r \varphi_{t}(X)}\left\|G_{i y}\right\|_{1 R_{t}} .
\end{aligned}
$$

But $\left|G_{i y}\right|=\beta_{1}\left(R_{t}|g|\right)$ which implies

$$
\int_{\Omega_{2}} R_{1}\left(\left|G_{i y}\right|\right) d \nu=\int_{\Omega_{2}} R_{t}(|g|) d \nu \leqslant 1
$$

since $\|g\|_{1 R_{t}} \leqslant 1$. Hence by definition,

Also

$$
\left\|G_{i y}\right\|_{1 R_{t}} \leqslant 1
$$

$$
\left\|\boldsymbol{F}_{i_{y}}\right\|_{y}=\left|\alpha_{i y} \varphi_{t}\left(\|f\|_{y}\right)\right|=a_{1}\left(p_{t}\left(\|f\|_{y}\right)\right)
$$


by (2.1.7), and therefore

$$
\int_{\Omega_{1}} \varphi_{1}\left(\left\|F_{i \nu}\right\|_{y}\right) d \mu=\int_{\Omega} \varphi_{t}\left(\|f\|_{y}\right) d \mu \leqslant 1
$$

since $\|f\|_{r_{\ell}(Y)}=1$. Hence

$$
\text { (2.1.19) } \quad\left\|F_{i j}\right\|_{r r_{1}(Y)} \leqslant 1 .
$$

But now (2.1.17), (2.1.18) and (2.1.19) give $I(i y) \leqslant 2 M_{1}$. Similarly $I(1+i y) \leqslant 2 M_{2}$.

It now follows from the three-line theorem,

and then by (2.1.3),

$$
I=I(t) \leqslant 2 M_{1}^{1-t} M_{2}^{t}
$$

$$
(2.1 .20) \quad\|\| T F\left\|_{X}^{r}\right\|_{S_{t}} \leqslant 2 M_{1}^{1-t} M_{2}^{t}
$$

But

$$
\|\| T f\left\|_{X}^{\dot{r}}\right\|_{1 S_{t}} \leqslant\|\| T f\left\|_{X}^{r}\right\|_{S_{t}}^{\circ} \leqslant 2\|\| T f\left\|_{X}^{r}\right\|_{1 S_{t}}
$$

(see [31]) and (2.1.20) becomes,

$$
\text { (2.1.21) } \quad\|T f\|_{r Q_{t}(X)}=\|\| T f\left\|_{X}^{r}\right\|_{1, S_{t}} \leqslant 4 . M_{1}^{1-t} M M_{2}^{t}
$$

for all $f \in \mathscr{L}(Y)$ such that $\|f\|_{r_{\ell}(Y)}=1$. Now let $f \in \mathscr{L}(Y)$ be arbitrary and let

$$
f^{\prime}=\frac{1}{\|f\|_{r \varphi_{l}(Y)}^{1 / r}} f
$$

Then $\left\|f^{\prime}\right\|_{r_{t}(Y)}=1$ and $\left\|T f^{\prime}\right\|_{Q_{Q_{t}}(X)} \leqslant 4 M_{1}^{1-t} M M_{2}^{t}$. But then

$$
\|T f\|_{r_{Q}(X)} \leqslant 4 \cdot M_{1}^{1-t} \cdot M_{2}^{t}\|f\|_{r^{\prime} \varphi_{t}(Y)} .
$$

Thus the theorem is completely proved. In the following the operator $T$ can be extended to the whole space under certain conditions.

CoroLmary 2.1.1. Let the hypothesis of Theorem 2.1.1 hold. If, in addition, $T$ is linear and $p_{i} \in \Delta_{2}, i=1,2$ then $T$ can be extended to all of $L^{\varphi_{t}(X)}$ with the same bound as in the above theorem.

CoroLrary 2.1.2. Let the hypothesis of Theorem 2.1.1 hold. If, in addition, $\varphi_{1}<\varphi_{2}$ (i.e. there exists constants $c_{1}, c_{2}$ and $u_{0}$ such that $p_{1}\left(c_{1} u\right)$ $\leqslant c_{2} \varphi_{2}(u)$ for $\left.u \geqslant u_{0} \geqslant 0\right) p_{1}, \varphi_{2}, Q_{1}, Q_{2} \in \Delta_{2}$, and $\mu\left(\Omega_{\perp}\right)<\infty$, then $T$ can be eatended to all of $L^{p_{t}(X)}$ with the some bound as in the theorem.

Proof. Since $\varphi_{1}<\varphi_{2}$, it follows that $\varphi_{1}<\varphi_{t}<p_{2}$ (see [24]). But then there exists constants $c_{1}, c_{2}$ such that

which implies

$$
\varphi_{1}\left(c_{1} u\right) \leqslant o_{2} \varphi_{t}(u) \quad \text { for } u \geqslant u_{0}
$$

or, equivalently

$$
\psi_{1}\left(c_{1}^{r} u^{r}\right) \leqslant c_{2} \psi_{t}\left(u^{r}\right) \quad \text { for } u \geqslant u_{0}
$$

$$
\psi_{1}\left(c_{1}^{\prime} u\right) \leqslant c_{2} \psi_{t}(u) \quad \text { for } u \geqslant u_{0}^{1 / r}
$$

and so $\psi_{1}<\psi_{t}$. But since $\mu\left(\Omega_{1}\right)<\infty$ there exists a constant $q$ (depending on $\mu\left(\Omega_{1}\right), u_{0}$ and the $p$ 's and $Q$ 's) such that

$$
\|f\|_{I_{\psi_{1}}} \leqslant q\|f\|_{1 q_{t}} \text { for all } f \in L^{\psi_{t}} \text { (see [14]); }
$$

or equivalently

$$
\|f\|_{r_{1}(Y)} \leqslant q\|f\|_{r \varphi_{t}(Y)} \quad \text { for all } f \in L^{\varphi_{t}(Y)} .
$$

Let $f \in L^{\varphi_{t}(Y)}$. Since $\varphi_{1} \in \Delta_{2}$, there exists $f_{n} \in \mathscr{L}(Y)$ such that

$$
\left\|f-f_{n}\right\|_{r \varphi_{t}(Y)} \rightarrow 0 \text {. }
$$

Since $\varphi_{1}<\varphi_{t}$, and $\mu(\Omega)<\infty, L^{\varphi_{t}(X)} \subset L^{\varphi_{1}(Y)}$ and so $f, f_{n} \in L^{\varphi_{1}(Y)}$. Consider

by (2.1.23). Therefore

$$
\begin{aligned}
\|\| T f\left\|_{X}-\right\| T f_{n}\left\|_{X}\right\|_{r Q_{1}} & \leqslant\left\|T\left(f-f_{n}\right)\right\|_{X}\left\|_{r Q_{1}}=\right\| T\left(f-f_{n}\right) \|_{r_{Q_{1}(X)}} \\
& \leqslant M_{1}\left\|f-f_{n}\right\|_{r r_{1}(Y)} \leqslant M_{1} q\left\|f-f_{n}\right\|_{r q_{t}(Y)}
\end{aligned}
$$

$$
\lim _{n \rightarrow \infty}\|\| T f\left\|_{X}-\right\| T f_{n}\left\|_{X}\right\|_{r_{Q_{1}}} \leqslant M_{1} q \lim _{n \rightarrow \infty}\left\|f-f_{n}\right\|_{r \varphi_{t}(Y),}=0 .
$$

Since $Q_{1} \in \Delta_{2}$, it follows that

$$
\lim _{n \rightarrow \infty} \int_{\Omega_{2}}\left(\|T f\|_{X}-\left\|T f_{n}\right\|_{X}\right) d v=0
$$

Consequently, there exists a subsequence $\left\{f_{n k}\right\}$ such that $Q_{1}\left(\|T f\|_{X}-\right.$ $\left.-\left\|T f_{n k}\right\|_{X}\right) \rightarrow 0$, a.e., and this implies $\left\|T f_{n k}\right\|_{X} \rightarrow\|T f\|_{X}$ a.e. since $Q_{1}$ is continuous. Since $\left\|f-f_{n}\right\|_{r \varphi_{t}(Y)} \rightarrow 0$ it follows that $\left\|f_{n k}\right\|_{r \varphi_{t}(Y)} \rightarrow\|f\|_{r \varphi_{t}(Y)}$. So consider

$$
\begin{aligned}
\int_{\Omega_{1}} Q_{t}\left(\frac{\|T f\|_{X}}{\left(4 M_{1}^{1-t} M_{2}^{t}\|f\|_{r \varphi_{t}(Y)}\right)^{1 / r}}\right) d v & =\int_{\Omega_{1}} \lim _{n_{k} \rightarrow \infty} Q_{t}\left(\frac{\left\|T f_{n k}\right\|_{X}}{\left(4 M_{1}^{1-t} M_{2}^{t}\left\|f_{n k}\right\|_{r \varphi_{t}(Y)}\right)^{1 / r}}\right) d v \\
& \leqslant \varliminf_{n_{k} \rightarrow \infty} \int_{\Omega_{1}} Q_{t}\left(\frac{\left\|T f_{n k}\right\|_{X}}{\left(4 M_{1}^{1-t} M_{2}^{t}\left\|f_{n k}\right\|_{r \varphi_{t}(Y)}\right)^{1 / r}}\right) d v \\
& \leqslant 1
\end{aligned}
$$

by Fatou's lemma, the fact that $Q_{t} \in \Delta_{2}$, and Theorem 2.1.1. So by definition

$$
\|T f\|_{r_{t}(X)} \leqslant 4 M_{1}^{1-t} M_{2}^{t}\|f\|_{r \varphi_{t}(X)} .
$$

An extension of the theorem in an infinite measure space can be given if the hypothesis is strengthened. This will be presented in the following proposition. 
Proposition 2.1.1. Let the hypothesis of Theorem 2.1.1 hold, with $\varphi_{1}<\varphi_{2}$ and $\varphi_{1}, \varphi_{2}, Q_{1}, Q_{2} \in \Delta_{2}$. If, in addition, there exists constants $K_{1}, K_{2}$ and $u_{1}$ such that

$$
\text { (2.1.25) } \quad \varphi_{2}\left(K_{1} u\right) \leqslant K_{2} \varphi_{t}(u) \text { for } u \leqslant u_{1} \text {, }
$$

then $T$ can be extended to all of $L^{\varphi_{t}(T)}$ with the same bound.

Proof. It follows from $p_{1}<p_{2}$ that $p_{1}<p_{t}<\varphi_{2}$, so that, there exist constants $K_{3}, K_{4}, u_{0}$ such that

$$
\varphi_{1}\left(K_{3} u\right) \leqslant K_{4} \varphi_{l}(u) \quad \text { for } u \geqslant u_{0}
$$

Let $f \in L^{\varphi_{t}(X)}$ and write $f=f_{m}^{\prime}+f_{m}^{\prime \prime}$, where

$$
f_{m}^{\prime}(\omega)=f(\omega) \quad \text { if }\|f(\omega)\|_{Y} \leqslant m u_{0}
$$

and 0 otherwise. Hence

$$
\left\|f_{m}^{\prime \prime}\right\|_{Y}>m u_{0} \quad \text { or }=0
$$

and

$$
\begin{gathered}
\left\|f_{m}^{\prime \prime}\right\|_{r \varphi_{l}(Y)} \rightarrow 0 \quad \text { as } m \rightarrow \infty, \text { since } \varphi_{t} \in \Delta_{2} . \\
E_{m}=\left\{\omega:\left\|f_{m}^{\prime}(\omega)\right\|_{Y} \geqslant \frac{u_{1}}{m}\right\} .
\end{gathered}
$$

Then $\mu\left(E_{m}\right)<\infty$ and if $g_{m}=f_{m}^{\prime} \chi_{E_{m}}, g_{m}$ is bounded and has finite support. Therefore there exists $f_{m} \epsilon \mathscr{L}(Y)$ such that

$$
\left\|f_{m}-g_{m}\right\|_{Y}<\frac{u_{1}}{m} \quad \text { for all } \omega
$$

and $f_{m}=0$ where $g_{m}=0$. But then

$$
\left\|f_{m}-f_{m}^{\prime}\right\|_{Y}<\frac{u_{1}}{m} \quad \text { for all } \omega
$$

since $\left\|f_{m}^{\prime}\right\|_{Y}<\frac{u_{1}}{m}$ outside $\mathbb{E}_{m}$. Now consider

(2.1.31) $\quad\left|\|T f\|_{X}-\left\|T f_{m}\right\|_{X}\right| \leqslant\left\|T\left(f-f_{m}\right)\right\|_{X} \leqslant\left\|T\left(f_{m}^{\prime}-f_{m}^{\prime}\right)\right\|_{X}+\left\|T f_{m}^{\prime \prime}\right\|_{X}$.

The idea is to show that there exists a subsequence such that $\mid\|T f\|_{x^{-}}$ $-\left\|T f_{m j}\right\|_{X} \mid \rightarrow 0$. Then as in Corollary $2.1 .2, T$ can be extended to all of $L^{\varphi_{t}(Y)}$ with the same bound. If follows from (2.1.25) and (2.1.30) that $f_{m}-f_{m}^{\prime} \in L^{\varphi_{2}(\bar{T})}$. So by hypothesis,

$$
\left\|T\left(f_{m}-f_{m}^{\prime}\right)\right\|_{r Q_{2}(X)} \leqslant M M_{1}\left\|f_{m}-f_{m}^{\prime}\right\|_{r r_{2}(X)} .
$$

Let $A_{k}=\left\{\omega:\|f(\omega)\|_{Y} \geqslant 1 / k\right\}$ and pick $k$ such that for $\varepsilon>0$ arbitrary,

$$
\int_{\mathcal{A}_{k}^{c}} \varphi_{t}\left(\|f\|_{Y}\right) d \mu<\varepsilon
$$

(this can be done since $\left.\int_{\Omega_{1}} \varphi_{t}\left(\|f\|_{Y}\right) d \mu<\infty\right)$.
Then

$$
\begin{aligned}
\int_{\Omega_{1}} \varphi_{t}\left(\left\|f_{m}-f_{m}^{\prime}\right\|_{Y}\right) d \mu= & \int_{A_{K}} \varphi_{t}\left(\left\|f_{m}-f_{m}^{\prime}\right\|_{Y}\right) d \mu \\
& +\int_{\mathcal{A}_{k}^{c}} \varphi_{t}\left(\left\|f_{m}-f_{m}^{\prime}\right\|_{Y}\right) d \mu .
\end{aligned}
$$

But for $m$ large enough, $f_{m}=0$ and $f_{m}^{\prime}=f$ on $A_{k}^{c}$. So for large $m$,

$$
\begin{aligned}
\int_{\Omega_{I}} \varphi_{t}\left(\left\|f_{m}-f_{m}^{\prime}\right\|_{Y}\right) d \mu & =\int_{A_{k}} \varphi_{t}\left(\left\|f_{m}-f_{m}^{\prime}\right\|_{Y}\right) d \mu+\int_{A_{k}^{c}} \varphi_{t}\left(\|f\|_{Y}\right) d \mu \\
& \leqslant \varphi_{t}\left(\frac{u_{1}}{m}\right) \mu\left(A_{k}\right)+\varepsilon .
\end{aligned}
$$

But $\mu\left(\dot{A}_{k}\right)<\infty$ and so

$$
\lim _{m \rightarrow \infty} \int_{\Omega_{1}} \varphi_{t}\left(\left\|f_{m}-f_{m}^{\prime}\right\|_{Y}\right) d \mu \leqslant \lim _{m \rightarrow \infty} \varphi_{t}\left(\frac{u_{1}}{m}\right) \mu\left(A_{k}\right)+\varepsilon=\varepsilon
$$

because $\varphi_{t}$ is continuous and $\varphi_{t}(0)=0$. Since $\varepsilon>0$ was arbitrary,

$$
\int_{\Omega_{1}} \varphi_{t}\left(\left\|f_{m}-f_{m}^{\prime}\right\|_{Y}\right) d \mu \rightarrow 0
$$

But by (2.1.25) and (2.1.30)

(2.1.33) $\quad \lim _{m \rightarrow \infty} \int_{\Omega_{1}} \varphi_{2}\left(K_{1}\left\|f_{m}-f_{m}^{\prime}\right\|_{Y}\right) d \mu \leqslant K_{2} \lim _{m \rightarrow \infty} \int_{\Omega_{1}} \varphi_{t}\left(\left\|f_{m}-f_{m}^{\prime}\right\|_{Y}\right) d \mu=0$.

It follows, since $\varphi_{2} \in \Delta_{2}$, that $\left\|f_{m}-f_{m}^{\prime}\right\|_{r \varphi_{2}(X)} \rightarrow 0$, and so by (2.1.32) $\lim _{m \rightarrow \infty}\left\|T\left(f_{m}-f_{m}^{\prime}\right)\right\|_{r Q_{2}(X)}=0$. But then there exists a subsequence such that

$$
\lim _{m_{k} \rightarrow \infty}\left\|T\left(f_{m_{K}}-f_{m_{K}}^{\prime}\right)\right\|_{X}=0 \quad \text { a.e. }
$$

By (2.1.26) and (2.1.28)

$$
\lim _{m \rightarrow \infty} \int_{\Omega_{1}} \varphi_{1}\left(K_{3}\left\|f_{m}^{\prime \prime}\right\|_{Y}\right) d \mu \leqslant K_{4} \lim _{m \rightarrow \infty} \int_{\Omega_{1}} \varphi_{t}\left(\left\|f_{m}^{\prime \prime}\right\|_{Y}\right) d \mu=0
$$

since $\left\|f_{m}^{\prime \prime}\right\|_{r \varphi_{t}(X)} \rightarrow 0$ and $\varphi_{t} \in \Delta_{2}$. Hence $\lim _{m \rightarrow \infty}\left\|f_{m}^{\prime \prime}\right\|_{r \varphi_{1}(Y)}=0$ and therefore,

$$
\lim _{m \rightarrow \infty}\left\|T f_{m}^{\prime \prime}\right\|_{r Q_{1}(X)} \leqslant M_{1} \lim _{m \rightarrow \infty}\left\|f_{m}^{\prime \prime}\right\|_{r \varphi_{1}(Y)}=0 .
$$

\footnotetext{
2 - Studia Mathematica XLIIr.2
} 
But then there exists another subsequence such that

$$
\lim _{m_{l} \rightarrow 0}\left\|T f_{m_{l}}^{\prime \prime}\right\|_{X}=0 \quad \text { a.e. }
$$

since $Q_{1} \in \Delta_{2}$. But the equations (2.1.34), (2.1.35) and (2.1.31) imply there exists a subsequence such that

$$
\lim _{m_{j} \rightarrow \infty}\left|\|T f\|_{X}-\left\|T f_{m_{j}}\right\|_{X}\right|=0 \text { a.e. }
$$

This completes the proof of the proposition.

2.2. Interpolation in Hardy-Orliez spaces. In this section, the convexity theorem for linear and sublinear operators on Hardy-Orlicz spaces will be given.

Proposinton 2.2.1. Let $\varphi_{i}, Q_{i}, \varphi_{t}, Q_{t}$ and $r$ be as in Theorem 2.1.1. In addition let $Q_{i}, \varphi_{i} \in \Delta_{2}$. If $T$ is a linear operator such that $T: H_{\varphi_{i}} \rightarrow H_{Q_{i}}$ $(i=1,2)$ and

$$
\|T F\|_{r H_{Q_{i}}} \leqslant M_{i}\|F\|_{r H \varphi_{i}} \text { for all } F \in H_{\varphi_{i}}
$$

then $T: H_{Q_{t}} \rightarrow H_{\varphi_{t}}$ with

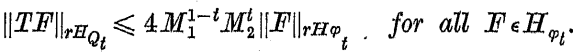

Proof. Let $\operatorname{Pr}(\theta-t)$ be the Poisson kernel and define for $f$, continuous on $[0,2 \pi]$,

$$
P f\left(r \theta^{i \theta}\right)=\frac{1}{2 \pi} \int_{0}^{2 \pi} \operatorname{Pr}(\theta-t) f(t) d t, \quad 0 \leqslant r<1 .
$$

Then the operator $P$ is linear and by Lemma 1.2.1, $P f \in N^{1}$. Since $f$ is continuous, it follows from Theorem 1.2.3 that $P f \in H_{\varphi}$, for any log-convex $\varphi$-function $\varphi$, and by Theorem 1.2.4

$$
\|P f\|_{H \varphi}=\|f\|_{\varphi} .
$$

If $\varphi(u)=\psi\left(u^{r}\right), 0<r \leqslant 1, \psi$ convex, then

$$
\|P f\|_{r H \varphi}=\|f\|_{r \varphi} \text {. }
$$

For $F \in H_{\varphi}$, let $f(\cdot)=F\left(e^{i \cdot}\right)$. Then by Theorem 1.2.4, $f \in L^{\varphi}(0,2 \pi)$ and $\left\|F^{\prime}\right\|_{H_{\varphi}}=\|f\|_{\varphi}$. Define

$$
R F(\cdot)=F^{\prime}\left(e^{i \cdot}\right) .
$$

Then $R: H_{\varphi} \rightarrow L_{\varphi}, R$ is linear, and

$$
\|R F\|_{\varphi}=\|F\|_{H_{\varphi}} \cdot
$$

Let $f$ be continuous and define $T^{*}$ by

$$
T^{*}(f)=R T P(f)
$$

then $T^{*}$ is well defined, linear, and $T^{*}: C(0,2 \pi) \rightarrow L^{Q_{i}}(0,2 \pi)(i=1,2)$ with

$$
\begin{aligned}
\left\|T^{*} f\right\|_{r Q_{i}} & =\|R T P f\|_{r Q_{i}}=\|T P(f)\|_{r B_{Q_{i}}} \\
& \leqslant M_{i}\|P f\|_{r H_{\varphi_{i}}}=M_{i}\|f\|_{r \varphi_{i}} \quad(i=1,2),
\end{aligned}
$$

by (2.2.4) (2.2.5) and the hypothesis. Since continuous functions are dense in $L^{q_{i}} T^{*}$ can be extended to all of $L^{\varphi_{i}}(0,2 \pi)$, preserving the bounds (2.2.8). But now the hypotheses of Theorem 2.1.1 are satisfied, and so $T^{*}: L^{q_{t}} \rightarrow L^{Q_{t}}$ with

$$
\text { (2.2.9) } \quad\|T f\|_{r Q_{t}} \leqslant 4 M_{1}^{1-t} M_{2}^{t}\|f\|_{r \varphi_{t}} \text {. }
$$

Let $F(z)$ be a polynomial. Then by [13], p. 33 there exists a continuous function $f$ such that

But then,

$$
F\left(r e^{i \theta}\right)=P f=\frac{1}{2 \pi} \int_{0}^{2 \pi} \operatorname{Pr}(\theta-t) f(t) d t .
$$

$$
\begin{aligned}
\text { (2.2.10) } \quad\|T F\|_{r H_{Q_{t}}} & =\|T P f\|_{r H_{Q_{t}}}=\|R T P f\|_{r Q_{t}}=\left\|T^{*} f\right\|_{r Q_{t}} \leqslant 4 M_{1}^{1-t} M_{2}^{t}\|f\|_{r \varphi_{t}} \\
& =4 M_{1}^{1-t} M_{2}^{t}\|P f\|_{r H_{\varphi_{t}}}=4 M_{1}^{1-t} M_{2}^{t}\left\|F^{H}\right\|_{r H_{\varphi_{t}}} .
\end{aligned}
$$

Since polynomials are dense in $H_{\varphi_{t}}\left(\varphi_{t} \in \Delta_{2}\right)$ and $T$ is linear $T$ can be extended to all of $H_{\varphi_{t}}$ with the same bound.

Proposimion 2.2.2. Let the hypothesis of Proposition 2.2.1 hold. If, instead, $T$ is sublinear and $\varphi_{1}<\varphi_{2}$, then the conclusion of Proposition 2.2.1 holds.

Proof. In Theorem 2.1.1, it is only necessary for $T$ to be defined on the continuous functions for the conclusion to hold. In this case, the proof of Proposition 2.2.1 is valid up to the point where $T$ is defined on all polynomials. But since $\varphi_{1}<\varphi_{2}$ and the measure is finite, $T$ can be extended to all of $H_{\varphi_{t}}$ with the same bound by a method similar to the proof of Corollary 2.1.2.

\section{INTERPOLATION WITH FACTORS}

In this section the interpolation theorem with factors is proven for sublinear operators on generalized Orlicz spaces. The theorem is similar to Theorem 2.1.1, but with the hypothesis that $\varphi_{i}, Q_{i}^{\prime} \mathrm{s}$ are $\Delta_{2}$ functions and the functions are not $B$-space valued (because of the factors). With these conditions the previous theorem is a special case of this theorem but the proofs are interesting enough for both to be included. All the known theorems on interpolation with change of measures can be shown to be special cases of these theorems. A deduction of this will be given later. 
3.1. Interpolation with factors in generalized Orlicz spaces. The following theorem is proved in [15].

THeOREM 3.1.1. Let $\varphi_{1}, \varphi_{2}$ be convex $p$-functions and

$$
\varphi_{t}^{-1}(\omega)=\left(\varphi_{1}^{-1}(\omega)\right)^{1-t}\left(\varphi_{2}^{-1}(\omega)\right)^{t} .
$$

Let $(\Omega, \Sigma, \mu)$ be a measure space and $u, v$ be measurable funotions. Let $a=u^{1-t} v^{t}$. Also let $\varphi_{i}=\psi_{i}\left(u^{r}\right), \psi_{i}$ convex, $0 \leqslant r<1$. Then for each $f$ such that $u f \epsilon L^{\varphi_{1}}$ and of $\epsilon L^{\varphi_{2}}$, one has af $\epsilon L^{\varphi_{t}}$. In fact

$$
\|a f\|_{r \varphi_{t}} \leqslant 4\|u f\|_{r \varphi_{1}}^{1-t}\|v f\|_{r \varphi_{2}}^{t} .
$$

This leads to the main result of this section:

THEOREM 3.1.2. Let $\varphi_{i}, Q_{i}(1=1,2)$ be $\Delta_{2}$-funotions such that $\varphi_{i}(u)=\psi^{\tilde{\nu}}\left(u^{r_{i}}\right)$ and $Q_{i}(u)=S_{i}^{\tilde{r}}\left(u^{s_{i}}\right)$ with $0<r_{i}, s_{i}, \leqslant 1$ and $\psi_{i}^{\tilde{i}}, S_{i}^{\tilde{i}}$ convex. Let $u_{1}, u_{2}$ be non-negative measurable functions on a measure space $(\Omega, \Sigma, \mu)$ such that $u_{i} \chi_{E} \in L^{\varphi_{i}}\left(\Omega_{1}, \Sigma_{1}, \mu\right)$ for $E \in \Sigma$, with $\mu(E)<\infty$. Also let $k_{1}, k_{2}$ be non-negative measurable functions on a measure space $\left(\Omega_{2}, \Sigma_{2}, v\right)$. Let $T$ be a sublinear mapping of $L^{\varphi_{i}}\left(\Omega_{1}, \Sigma_{2}, \mu\right)$ into $L^{Q_{i}}\left(\Omega_{2}, \Sigma_{2}, v\right)$ such that for some $0<r \leqslant \min \left\{r_{1}, r_{2}, s_{1}, s_{2}\right\}$

$$
\left\|k_{1} T(f)\right\|_{r_{1}} \leqslant M_{1}\left\|f u_{1}\right\|_{r \varphi_{1}}
$$

for all $f$ such that $u_{1} f \in L_{p_{1}}$, and

$$
\left\|k_{2} T(f)\right\|_{r_{2}} \leqslant M_{2}\left\|f u_{2}\right\|_{r \varphi_{2}}
$$

for all $f$ such that $u_{2} f \in L_{p_{2}}$. If $\left(\varphi_{t}^{-1}\right)=\left(\varphi_{t}^{-1}\right)^{1-t}\left(\varphi_{2}^{-1}\right)^{t}, Q_{t}^{-1}=\left(Q_{1}^{-1}\right)^{1-t}\left(Q_{2}^{-1}\right)^{t}$, $k_{t}=k_{1}^{1-t} k_{2}^{t}$, and $u_{t}=u_{1}^{1-t} u_{2}^{t}$, then

$$
\left\|k_{t} T(f)\right\|_{r_{Q_{t}}} \leqslant 4 . M_{1}^{1-t} M_{2}^{t}\left\|f u_{t}\right\|_{r_{t}}
$$

for all $f \in \mathscr{L}_{1}$, the class of simple functions on $\left(\Omega_{1}, \Sigma_{1} 1 \mu\right)$.

Proof. Let $\psi_{i}, S_{i}, \psi_{t}$ and $S_{t}$ be defined as in Theorem 2.1.1. Assumptions will be made on $u_{1}$ and $u_{2}$ that will be removed later. So assume $u_{1}, u_{2} \geqslant \varepsilon_{1}>0$. Let $f \in \mathscr{L}_{1}$ be such that $\|f\|_{r \varphi_{t}}=1$ and $g \in \mathscr{L}_{2}$, (the simple functions on $\left.\left(\Omega_{2}, \Sigma_{2}, \nu\right)\right)$ be such that $\|g\|_{1 R_{t}} \leqslant 1$, where $R_{t}$ is as in Theorem 2.1.1. Consider

Let

$$
I=\int_{\Omega_{2}}\left|\hbar_{t_{t}}\right|^{r}\left|T\left(u_{t}^{-1} f\right)\right|^{r}|g| d v
$$

$$
f=\sum_{j=1}^{m_{1}}\left|a_{j}\right| e^{i \theta_{j}} \chi_{F_{j}}, \quad g=\sum_{l=1}^{m_{2}}\left|b_{l}\right| e^{i \theta^{\prime} l} \chi_{G_{l}}
$$

and define

$$
F_{z}=\alpha_{z}\left(\varphi_{t}|f|\right) e^{i \theta}=\sum_{j=1}^{m_{1}} \alpha_{z}\left(\varphi_{t}\left|a_{j}\right|\right) e^{i \theta_{j}} \chi_{F_{j}} ;
$$

and

$$
G_{z}=\beta_{z}\left(R_{l}(|g|)\right) e^{i \theta \prime}=\sum_{l=1}^{m_{2}} \beta_{z}\left(R_{t}\left|b_{l}\right|\right) e^{i \theta_{l}} \chi_{G_{l}}
$$

where $\alpha_{z}$ and $\beta_{z}$ are defined in Theorem 2.1.1. Let $k_{z}=k_{1}^{\mathrm{i}-z} k_{2}^{z}$ and $v_{z}$ $=\left(u_{1}^{-1}\right)^{1-z}\left(u_{2}^{-1}\right)^{z}$. Note that

(3.1.5) $\left|v_{z}\right|=\left|u_{1}^{-1}\right|^{1-x}\left|u_{2}^{-1}\right|^{x} \leqslant\left(\frac{1}{\varepsilon_{1}}\right)^{1-x}\left(\frac{1}{\varepsilon_{1}}\right)^{x}=\frac{1}{\varepsilon_{1}}, \quad(\chi=\operatorname{Re} z)$.

Now define

$$
I(z)=\int_{\Omega_{2}}\left|k_{z}\right|^{r}\left|T\left(v_{z} F_{z}\right)\right|^{r}\left|G_{z}\right| d \nu
$$

The plan, as in Theorem 2.1.1, is to show that $I(z)$ satisfies the hypothesis of the three-line theorem for subharmonic functions, since $I(t)=I$. The desired result will follow easily from this theorem. $I(z)$ can be simplified. Let

$$
\lambda_{z}^{l}=\sum_{j=1}^{m_{2}} \beta_{z}^{1 / r}\left(R_{t}\left(\left|b_{l}\right|\right)\right) \alpha_{z}\left(\varphi_{t}\left(\left|a_{j}\right|\right)\right) e^{i\left(\theta_{j}+\theta_{l}^{\prime}\right)} \chi_{F_{j}}
$$

then $\lambda_{z} \epsilon \mathscr{L}_{1}$ for each $z$. Let $\gamma_{z}^{l}=v_{z} \lambda_{z}^{l}$. Then

'This is true since

$$
u_{1} \gamma_{z}^{l} \in L^{\varphi_{1}} \text { and } u_{2} \gamma_{z}^{l} \epsilon L^{\varphi_{2}} \text { : }
$$

$$
u_{1} \gamma_{z}^{l} \leqslant u_{1}\left|\gamma_{z}\right|=u_{1}\left|v_{z}\right|\left|\gamma_{z}\right| \leqslant \frac{u_{1}}{\varepsilon_{1}}\left|\gamma_{z}\right|
$$

But $\frac{1}{\varepsilon_{1}}\left|\gamma_{g}\right| \epsilon \mathscr{L}_{1}$ and so by hypothesis $\frac{u_{1}}{\varepsilon_{1}}\left|\gamma_{z}\right| \epsilon L^{\varphi_{1}}\left(u_{1} \chi_{E} \epsilon L^{\varphi_{1}}\right.$ for all $E \epsilon \Sigma$ such that $\mu(E)<\infty$ clearly implies $u_{1} f \in L^{\varphi_{1}}$ for all simple functions $f$ ). Similarly $u_{2} \gamma_{z} \epsilon L^{\varphi_{2}} . I(z)$ can now be written,

$$
I(z)=\sum_{l=1}^{m_{2}} \int_{\sigma_{l}}\left|k_{z}\right|^{r}\left|T\left(\gamma_{z}\right)\right|^{r} d \nu=\sum_{l=1}^{m_{2}} \Gamma_{l}(z) .
$$

From now on, the proof proceeds in steps.

Step 1. $\Gamma_{l}(z)$ is subharmonic in $0<x<1$. For this it is necessary to show $\log \left|T\left(\gamma_{z}^{l}\right)\right|$ is subharmonic. For this it is sufficient to show $e^{h(z)}\left|T\left(\gamma_{z}\right)\right|$ is subharmonic for any harmonic $h$ in $0<x<1$. The proof is similar to that of Theorem 2.1.1, to which it reduces if $u_{1}=1=v_{1}$. There are, however, some complications since these functions are not constants. Since a function is subharmonic in a region if it is subharmonic in a neighborhood of each point, fix $z$, take $\varrho>0$, and let $z_{1}, \ldots, z_{p}$ be a set of 
points equally spaces on the circle of radius $\varrho$ about $z$. Then it is sufficient to show

(3.1.10)

$$
e^{h(z)} \Gamma_{l}(z) \leqslant \frac{1}{2 \pi} \int_{0}^{2 \pi} e^{h\left(z+e^{i \theta}\right)} \Gamma_{l}\left(z+\varrho e^{i \theta}\right) d \theta
$$

Let $H(z)$ be the analytic function whose real part is $h(z)$, and

(3.1.11)

then $\gamma_{z}^{* l}=v_{z} \lambda_{z}^{* l}$ and

$$
\text { (3.1.12) } \quad u_{1} \gamma_{z}^{* l} \in L^{\Phi_{1}}, \quad u_{2} \gamma_{z}^{* l} \in L^{m_{2}}
$$

since $h(z)$ is bounded in $\{\xi:|z-\xi| \leqslant \varrho\}$. (The proof is the same as for (3.1.8). It is elear that $\gamma_{z}^{* l}$ is analytic for each $\omega \in \Omega_{1}$ and therefore

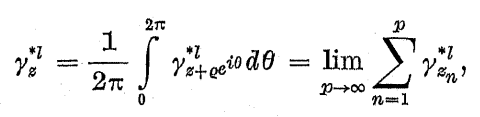

where $z_{n}=\varrho e^{i \Delta \theta_{n}}$ and $\Delta \theta_{n}=\frac{1 \pi}{p}$. By the sublinearity of $T$,

$$
\begin{aligned}
\left|T\left(\gamma_{z}^{* l}\right)\right| & =\left|T\left(\gamma_{z}^{* l}\right)\right|-\left|T\left(1 / p \sum_{n=1}^{p} \gamma_{z_{n}}^{* l}\right)\right|+\left|T\left(1 / p \sum_{n=1}^{p} \gamma_{z_{n}}^{* l}\right)\right| \\
& \leqslant\left|T\left(\gamma_{z}^{* l}-1 / p \sum_{n=1}^{p} \gamma_{z_{n}}^{* l}\right)\right|+\left|T\left(1 / p \sum_{n=1}^{p} \gamma_{z_{n}}^{* l}\right)\right| \\
& \leqslant\left|T\left(\gamma_{z}^{* l}-1 / p \sum_{n=1}^{p} \gamma_{z_{n}}^{* l}\right)\right|+1 / p \sum_{n=1}^{p}\left|T \gamma_{z_{n}}^{* l}\right| .
\end{aligned}
$$

The plan is to show $\left|T\left(\gamma_{z}^{* l}\right)\right|$ is subharmonic for each $\omega$. For this it is sufficient to show that there exists a subsequence such that

$$
\lim _{p_{k} \rightarrow \infty}\left|T\left(\gamma_{z}^{*}-1 / p_{l} \sum_{n=1}^{p_{k}} \gamma_{z}^{*}\right)\right|=0 \quad \text { a.e. }
$$

for, (3.1.14) becomes

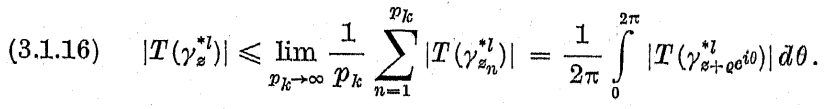

The first step is to show

since

$$
\lim _{p \rightarrow \infty}\left\|u_{1}\left(\gamma_{z}^{*}-1 / p \sum_{n=1}^{p} \gamma_{z_{n}}^{* l}\right)\right\|_{r q_{1}}=0
$$

(3.1.18) $\left\|k_{1} T\left(\gamma_{z}^{* l}-1 / p \sum_{n=1}^{p} \gamma_{z_{n}}^{*}\right)\right\|_{r Q_{1}} \leqslant M_{1}\left\|u_{1}\left(\gamma_{z}^{* l}-1 / p \sum_{n=1}^{p} \gamma_{z_{n}}^{* l}\right)\right\|_{r p_{1}}$ by hypothesis and (3.1.12). But since $\varphi_{1} \epsilon \Delta_{2}$, (3.1.17) will follow: from

$$
\lim _{p \rightarrow \infty} \int_{\Omega_{1}} \varphi_{1}\left(u_{1}\left(\gamma_{z}^{* l}-1 / p \sum_{n=1}^{p} \gamma_{z_{n}}^{*} l\right)\right) d \mu=0 .
$$

Consider,

(3.1.20) $\left|\gamma_{z}^{*}\right| \leqslant \sum_{j=1}^{m_{1}} \mid \beta_{z}\left(\left.R_{t}\left(b_{l}\right)\right|^{1 / r}\left|\alpha_{z}\left(\varphi_{t}\left(\left|a_{j}\right|\right)\right)\right||| v_{q} \mid e^{h(s)} \chi_{F_{j}} \leqslant M^{\prime} \sum_{j=1}^{m_{1}} \chi_{F_{j}}\right.$

since $\left|\beta_{z}\right|,\left|\alpha_{z}\right|,\left(v_{z} \mid\right.$ and $e^{h(z)}$ are bounded for all $z \in\{\xi:|\xi-z| \leqslant \varrho\}$ and all $\omega \epsilon \Omega_{1}$. Therefore

$$
\begin{aligned}
\left|\gamma_{z}^{*}-1 / p \sum_{n=1}^{p} \gamma_{z_{n}}^{* l}\right| \leqslant & \left|\gamma_{z}^{* l}\right|+1 / p \sum_{n=1}^{p}\left|\gamma_{z_{n}}^{* z_{n}}\right| \\
\leqslant & M^{\prime} \sum_{j=1}^{m_{1}} \chi_{F_{j}}+1 / p \sum_{n=1}^{p} M^{\prime} \sum_{j=1}^{m_{1}} \chi_{F_{j}} \\
& =2 M^{\prime} \sum_{j=1}^{m_{1}} \chi_{F_{j}}:
\end{aligned}
$$

But $2 M^{\prime} \sum_{j=1}^{m_{1}} \chi_{F_{j}} \epsilon \mathscr{L}_{1}$ and so by hypothesis $u_{1}\left(2 M^{\prime} \sum_{j=1}^{m_{1}} \chi_{F_{j}}\right) \epsilon L^{\varphi_{1}}$ and therefore

$$
\varphi_{1}\left(u_{1}\left|\gamma_{z}^{* l}-1 / p \sum_{n=1}^{p} \gamma_{z_{n}}^{*}\right|\right) \leqslant \varphi_{1}\left(2 M \sum_{j=1}^{p} \chi_{F_{j}}\right) \epsilon L^{1},
$$

since $\varphi_{1} \in \Delta_{2}$. So by the Lebesque Dominated Convergence Theorem,

$$
\begin{aligned}
\lim _{p \rightarrow \infty} \int_{\Omega_{1}} \varphi_{1}\left(u_{1} \mid \gamma_{z}^{* l}-1 / p\right. & \left.\sum_{n=1}^{p} \gamma_{z_{n}}^{* l} \mid\right) d \mu \\
& =\int_{\Omega_{1}} \lim _{p \rightarrow \infty} \varphi_{1}\left(u_{1}\left|\gamma_{z}^{* l}-1 / p \sum_{n=1}^{p} \gamma_{z_{n}}^{*}\right|\right) d \mu=0
\end{aligned}
$$

(3.1.13) since $\varphi_{1}$ is continuous. Hence (3.1.17) holds, and therefore since $Q_{1} \in \Lambda_{2}$

$$
\lim _{p \rightarrow \infty} \int_{\Omega_{2}} Q_{1}\left(k_{1} T\left(\gamma_{z}^{* l}-\mathfrak{I} / p \sum_{n=1}^{p} \gamma_{z_{n}}^{* l}\right)\right) d v=0
$$

which implies the existence of a subsequence such that

$$
\left|T\left(\gamma_{z}^{* l}-1 / p_{k} \sum_{n=1}^{p_{k}} \gamma_{z_{n}}^{* l}\right)\right| \rightarrow 0 \quad \text { a.e. }
$$

So (3.1.15) holds and therefore $\left|T\left(\gamma_{z}^{* l}\right)\right|$ is subharmonic for each $\omega . \mathrm{Bu}$ (3.1.25) $\quad\left|T\left(\gamma_{z}^{* l}\right)\right|=\left|T\left(e^{H(z)} \gamma_{z}^{l}\right)\right|=\left|e^{H(z)}\right|\left|T\left(\gamma_{z}^{l}\right)\right|=e^{h(z)}\left|T\left(\gamma_{z}^{l}\right)\right|$ 
and so $\log \left|T\left(\gamma_{z}^{l}\right)\right|$ is subharmonic. Since $K_{z}$ is analytic, $\log \left|k_{z}\right|$ is subharmonic also. So if $\Lambda(z)=\left|k_{z}\right|^{r}\left|T\left(\gamma_{z}^{l}\right)\right|^{r}$,

$$
\text { (3.1.26) } \quad \log \Lambda(z)=r \log \left|k_{z}\right|+r \log \left|T\left(\gamma_{z}^{l}\right)\right|
$$

and therefore $\log \Lambda(z)$ is subharmonic. But then if $h(z)$ is any harmonic function, $e^{h(z)} \Lambda(z)$ is subharmonic, and so

$$
e^{h(z)} \Lambda(z) \leqslant \frac{1}{2 \pi} \int_{0}^{2 \pi} e^{h\left(z+e^{i \theta}\right)} \Lambda\left(z+\varrho e^{i \theta}\right) d \theta
$$

for each $\omega$. Hence,

$$
\begin{aligned}
e^{h(z)} \Gamma_{l}(z) & =\int_{\sigma_{l}} e^{h(z)} \Lambda(z) d \nu \leqslant \int_{\sigma_{l}}\left(\frac{1}{2 \pi} \int_{0}^{2 \pi} e^{h\left(z+e^{i \theta}\right)} \Lambda\left(z+\varrho e^{i \theta}\right) d \theta\right) d v \\
& =\frac{1}{2 \pi} \int_{0}^{2 \pi}\left(\int_{\sigma_{l}} e^{h\left(z+e^{i \theta}\right)} \Lambda\left(z+\varrho e^{i \theta}\right) d \nu\right) d \theta \\
& =\frac{1}{2 \pi} \int_{0}^{2 \pi} e^{h\left(z+e^{e i \theta}\right)} \Gamma_{l}\left(z+\varrho e^{i \theta}\right) d \theta
\end{aligned}
$$

It follows that $\log \Gamma_{l}(z)$ is subharmonic.

Step 2. $\Gamma_{l}(z)$ is continuous. Oonsider

(3.1.29) $\left|\Gamma_{l}(z+\Delta z)-\Gamma_{l}(z)\right|$

$$
\begin{aligned}
& \leqslant\left.\int_{\sigma_{l}}|| k_{z+\Delta z}\right|^{r}\left|T\left(\gamma_{z+\Delta z}^{l}\right)\right|^{r}-\left|k_{z}\right|^{r}\left|T\left(\gamma_{z}^{l}\right)\right|^{r} \mid d \nu \\
& \leqslant\left.\int_{\sigma_{l}}|| k_{z+\Delta z}|r| T\left(\gamma_{z+\Delta z}^{l}\right)\right|^{r}-\left|k_{z+\Delta z}\right|^{r}\left|T\left(\gamma_{z}^{l}\right)\right|^{r}+\left|k_{z+\Delta z}\right|^{r}\left|T\left(\gamma_{z}^{l}\right)\right|^{r}-\left|k_{z}\right|{ }^{r}\left|T\left(\gamma_{z}^{l}\right)\right|^{r} \mid d \nu \\
& \leqslant\left.\int_{\sigma_{l}}|| k_{z+\Delta z}\right|^{r}\left(\left|T\left(\gamma_{z+\Delta z}^{l}\right)\right|^{r}-\left|T\left(\gamma_{z}^{l}\right)\right|^{r}\right) d \nu+\int_{\sigma_{l}}\left|\left(\left|k_{z+\Delta z}\right|^{r}-\left|k_{z}\right|^{r}\right)\right|\left|T\left(\gamma_{z}^{l}\right)^{\gamma}\right| d \nu \\
& \leqslant \int_{\sigma_{l}}\left|k_{z+\Delta z}\right|^{r}\left|T\left(\gamma_{z+\Delta z}^{l}-\gamma_{z}^{l}\right)\right|^{r} d \nu+\int_{\sigma_{l}}\left(\left|k_{z+\Delta z}\right|-\left|k_{z}\right|\right)^{r}\left|T\left(\gamma_{z}^{l}\right)\right|^{r} d \nu
\end{aligned}
$$

by the sublinearity of $T$ and subadditivity of $w^{r}$. Note that for all $z=x+i y$ such that $0 \leqslant x \leqslant 1$,

(3.1.30) $\left|k_{z}\right|=\left|k_{1}^{1-z} k_{2}^{z}\right|=k_{1}^{1-x} k_{2}^{x} \leqslant \max _{x}\left\{k_{1}\left(\frac{k_{k_{2}}}{k_{1}}\right)^{\infty}\right\}=\max \left\{k_{1}, k_{2}\right\} \leqslant k_{1}+k_{2}$. Therefore, since $0<r \leqslant 1$ and $0 \leqslant \operatorname{Re}(z+\Delta z) \leqslant 1$,

(3.1.31) $\quad \int_{G_{l}}\left|k_{z+\Delta z}\right|^{r}\left|T\left(\gamma_{z+\Delta z}^{l}-\gamma_{z}^{l}\right)\right|^{r} d \nu$

$$
\begin{aligned}
& \leqslant \int_{G_{l}} k_{1}^{r}\left|T\left(\gamma_{z+\Delta z}^{l}-\gamma_{z}^{l}\right)\right|^{r} d v+\int_{\sigma_{l}} k_{2}^{r}\left|T\left(\gamma_{z+\Delta z}^{l}-\gamma_{z}^{l}\right)\right|^{r} d v \\
& \leqslant\left\|k_{1} T\left(\gamma_{z+\Delta z}^{l}-\gamma_{z}^{l}\right)\right\|_{r Q_{Q^{2}}}\left\|\chi_{G_{1}}\right\|_{R_{1}}^{0}+\left\|k_{2} T\left(\gamma_{z+\Delta z}^{l}-\gamma_{z}^{l}\right)\right\|_{Q_{2}}\left\|\chi_{G_{l}}\right\|_{R_{2}}^{o} \\
& \leqslant M_{1}\left\|u_{1}\left(\gamma_{z+\Delta z}^{l}-\gamma_{z}^{l}\right)\right\|_{r \varphi_{1}}\left\|\chi_{G_{l}}\right\|_{R_{1}}^{o}+M_{2}\left\|u_{2}\left(\gamma_{z+\Delta z}^{l}-\gamma_{z}^{l}\right)\right\|_{r_{2} p_{2}}\left\|\chi_{G_{l}}\right\|_{R_{2}}^{o}
\end{aligned}
$$

by hypothesis. It can be shown that $\left\|u_{i}\left(\gamma_{z+\Delta z}^{l}-\gamma_{z}^{l}\right)\right\|_{r \varphi_{i}} \rightarrow 0$ as $\Delta z \rightarrow 0$ by an argument similar to that used in Step 1 to show

$$
\left\|u_{1}\left(\gamma_{z}^{l}-1 / p \sum_{n=1}^{p} \gamma_{z_{n}}^{l}\right)\right\|_{r_{1}} \rightarrow 0
$$

using the Dominated Convergence Theorem. Hence, the first integral on the right of (3.1.29) $\rightarrow 0$ as $\Delta z \rightarrow 0$.

As for the second term of (3.1.29), consider

(3.1.32) $\quad \int_{G_{l}}\left(\left|k_{z+\Delta z}\right|-\left|k_{z}\right|\right)^{r}\left|T\left(\gamma_{z}^{l}\right)\right| d v \leqslant\left\|\left(\left|k_{z+\Delta s}\right|-\left|k_{z}\right|\right)^{r}\left|T\left(\gamma_{z}^{l}\right)\right|^{r}\right\|_{1 S_{1}}\left\|\chi_{G_{l}}\right\|_{R_{1}}^{\circ}$

$$
=\left\|\left(\left|k_{z+\Delta z}\right|-\left|k_{z}\right|\right)\left|T\left(\gamma_{z}^{l}\right)\right|\right\|_{r_{1}}\left\|\chi_{G_{z}}\right\|_{R_{1}}^{\circ} .
$$

The first term on the right can be shown to converge to zero as $\Delta z \rightarrow 0$, again using the Dominated Convergence Theorem. Hence the second integral on the right of (3.1.29) $\rightarrow 0$ as $\Delta z \rightarrow 0$, i.e.

$$
\lim _{\Delta z \rightarrow 0}\left|\Gamma_{l}(z+\Delta z)-\Gamma_{l}(z)\right|=0,
$$

and so $\Gamma_{l}(z)$ is continuous in $0 \leqslant x \leqslant 1$.

Step 3. $\Gamma_{l}(z)$ is bounded. Consider

$$
\begin{aligned}
& \int_{G_{l}}\left|k_{r}\right|^{r}\left|T\left(\gamma_{z}^{l}\right)\right|^{r} d \nu \leqslant \int_{G_{l}}\left(k_{1}^{r}+k_{2}^{r}\right)\left|T T\left(\gamma_{z}^{l}\right)\right|^{r} d \nu \\
& =\left.\int_{G_{l}} k_{1}^{r}\left|T\left(\gamma_{z}^{l}\right)^{r} d \nu+\int_{G_{l}} k_{2}^{r}\right| T\left(\gamma_{z}^{l}\right)\right|^{r} d v \\
& \leqslant\left\|k_{1} T\left(\gamma_{z}^{l}\right)\right\|_{r_{1}}\left\|\chi_{G_{l}}\right\|_{R_{1}}^{\circ}+\left\|k_{2} T\left(\gamma_{z}^{l}\right)\right\|_{r Q_{2}}\left\|\chi_{G_{l}}\right\|_{R_{2}}^{\circ} \\
& \leqslant M_{1}\left\|u_{1} \gamma_{z}^{l}\right\|_{r_{\varphi_{1}}}\left\|\chi_{G_{l}}\right\|_{R_{1}}^{\circ}+M_{2}\left\|u_{2} \gamma_{z}^{l}\right\|_{r_{2}}\left\|\chi_{G_{l}}\right\|_{R_{2}}^{\circ}
\end{aligned}
$$

by Holder's inequality and (3.1.30). But by (3.1.21) this becomes,

$$
\begin{aligned}
& \int_{\sigma_{l}}\left|k_{z}\right|^{r}\left|T\left(\gamma_{z}^{l}\right)\right|^{r} d \mu \\
& \leqslant M_{1} M^{\prime} \sum_{j=1}^{m_{1}}\left\|\chi_{F_{j}}\right\|_{r_{1}}\left\|\chi_{\sigma_{l}}\right\|_{R_{1}}^{\circ}+M_{1} M^{\prime} \sum_{j=1}^{m_{1}}\left\|\chi_{F_{j}}\right\|_{r r_{2}}\left\|\chi_{\sigma_{l}}\right\|_{R_{2}}^{\circ}<\infty .
\end{aligned}
$$

Hence $\Gamma_{l}(z)$ is bounded. Now, since $I(z)=\sum_{l=1}^{m_{2}} \Gamma_{l}(z)$. It follows that $I(z)$ is continuous and bounded in $0 \leqslant x \leqslant 1$, and $\log I(z)$ is subharmonic in $0<x<1$.

Step 4. $I(i y) \leqslant 2 M_{1}$ and $I(1+i y) \leqslant 2 M_{2}$. Consider

$$
\begin{aligned}
I(i y) & =\int_{\Omega_{2}}\left|k_{i y}\right|^{r}\left|T\left(v_{i j} F_{i y}\right)\right|^{r}\left|G_{i y}\right| d v \\
& \leqslant 2\left\|k_{1}^{r}\left|k_{1}^{-i y} k_{2}^{i y}\right|^{r}\left|T\left(v_{i y} F_{i y}\right)\right|^{r}\right\|_{1 S_{1}}\left\|G_{i y}\right\|_{1 R_{1}} \\
& =2 \| k_{1} T\left(u_{1}^{-1}\left(u_{1}^{i v} u_{2}^{-i y} F_{i y}\right)\left\|_{r Q_{1}}\right\| G_{i y} \|_{1 R_{1}}\right. \\
& \leqslant 2 M_{1}\left\|F_{i y}\right\|_{r r_{1}}\left\|G_{i y}\right\|_{1 R_{1}} .
\end{aligned}
$$


But $\left\|F_{i y}\right\|_{r \varphi_{1}} \leqslant 1$ and $\left\|G_{i y}\right\|_{1 R_{1}} \leqslant 1$, and so $I(i y) \leqslant 2 M_{1}$. Similarly $I(1+i y)$ $\leqslant 2 M_{2}$. Now in view of the three-line theorem,

$$
I=I(t) \leqslant 2 M_{1}^{1-t} M_{2}^{t} .
$$

So as in Theorem 2.1.1,

$$
\left\|k_{t} T\left(u_{t}^{-1} f\right)\right\|_{r_{o_{l}}} \leqslant 4 \cdot M_{1}^{1-t} M_{2}^{t}\|f\|_{r_{p_{t}}}
$$

for all $f \in \mathscr{L}_{1}$, and therefore

(3.1.37)

for all $f \in \mathscr{L}$.

Now the assumptions on $u_{1}, u_{2}$ will be removed. Let

(3.1.38),$u_{i}^{n}=u_{i}$ if $u_{i} \geqslant 1 / n$ and $u_{i}^{n}=1 / n$ otherwise $(i=1,2)$.

Then $u_{1}^{n}, u_{2}^{n} \geqslant 1 / n$ and $u_{i} \leqslant u_{i}^{n}$. But then $u_{i} f \leqslant u_{i}^{n} f$, and so by hypothesis,

$$
\left\|k_{i}(T f)\right\|_{r_{i}} \leqslant M_{i}\left\|u_{i} f\right\|_{r r_{i}} \leqslant M_{i}\left\|u_{i}^{n} f\right\|_{r p_{i}} .
$$

But since $u_{1}, u_{2} \geqslant 1 / n$, the above proof applies, and therefore for all $n$, (3.1.40)

$$
\left\|\bar{k}_{t} T(f)\right\|_{r_{Q_{t}}} \leqslant\left\|u_{t}^{n} f\right\|_{r_{t}}
$$

for each $f \in \mathscr{L}_{1}$ where $u_{t}^{n}=\left(u_{1}^{n}\right)^{1-t}\left(u_{2}^{n}\right)^{t}$. It is now necessary to show

Consider

$$
\lim _{n \rightarrow \infty}\left\|u_{t}^{n} f\right\|_{r_{t}}=\left\|u_{t} f\right\|_{r p_{t}} .
$$

$$
\left|u_{t}^{n} f-u_{t} f\right|=\left(u_{t}^{n}-u_{t}\right)|f| \leqslant\left(\dot{u}_{t}^{1}-u_{t}\right)|f| \leqslant\left(u_{t}^{1}+u_{t}\right)|f| \leqslant 2 u_{t}^{1}|f|
$$

since $u_{t}^{n}$ decreases with $n$. But $u_{1}^{1} f=u_{1} \chi_{A \perp} f+\chi_{A^{\prime}} c \in L^{\omega_{1}}$ where $A=\left\{\omega: u_{1}(\omega)\right.$

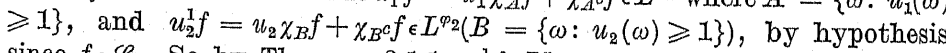
since $f \in \mathscr{L}_{1}$. So by Theorem 3.1.1, $u_{t}^{1} f \in L^{p} t$, and therefore

$$
p_{t}\left(\left|u_{t}^{n} f-u_{t} f\right|\right) \leqslant \varphi_{t}\left(2 u_{t}^{1}|f|\right) \in L^{1}
$$

and so by the Dominated Convergence Theorem,

$$
\lim _{n \rightarrow \infty} \int_{\Omega_{1}} p_{t}\left(\left|u_{t}^{n} f-u_{t} f\right|\right) d \mu=\int_{\Omega_{1}} \lim _{n \rightarrow \infty} \varphi_{t}\left(\left|u_{t}^{n} f-u_{t} f\right|\right) d \mu=0
$$

and so be the $\Delta_{2}$ condition,

$$
\left\|u_{t}^{n} f-u_{t} f\right\|_{r \varphi_{t}} \rightarrow 0 .
$$

Hence (3.1.40) holds and so for all $f \in \mathscr{L}_{1}$,

$$
\left\|k_{i_{t}} T(f)\right\|_{r_{Q_{t}}} \leqslant \lim _{n \rightarrow \infty}\left\|u_{t}^{n} f\right\|_{r_{r_{t}}}=\left\|u_{t} f\right\|_{r r_{t}} .
$$

- This completes the proof. here.

\section{FURTHER RESULTS}

A theorem on the interpolation of a smooth family of operators which generalizes Stein's result on an analytic family [29] is given in subsection 4.1. Subsection 4.2 deals with change of measures.

4.1. Smooth families of operators. Let $L\left(\Omega_{1}, \Sigma_{1}, \mu\right)$ denote the class of simple functions on a measure space $\left(\Omega_{1}, \Sigma_{1}, \mu\right)$ and $\mathscr{M}\left(\Omega_{2}, \Sigma_{2}, v\right)$ the class of measurable functions on a measure space $\left(\Omega_{2}, \Sigma_{2}, \nu\right)$.

DEFINITION 4.1.1. Suppose $\gamma_{z}(\omega)$ is a function of $z$ and $\omega$ such that $\gamma_{z}(\cdot) \in L\left(\Omega_{1}, \Sigma_{1}, \mu\right)$ for each $z$ and $\gamma \cdot(\omega)$ is analytic for each $\omega$. Then $\gamma_{z}(\omega)$ is called an analytic simple function on $\left(\Omega_{1}, \Sigma_{1}, \mu\right)$.

DEFINITION 4.1.2. A family of operators $T_{z}$ (depending on the complex parameter $z$ ), is called a smooth family if the following conditions hold:

(i) $T_{z}: L\left(\Omega_{1}, \Sigma_{1}, \mu\right) \rightarrow \mathscr{M}\left(\Omega_{2}, \Sigma_{2}, v\right)$ for each $z$.

(ii) If $\gamma_{z}(\omega)$ is an analytic simple function on $\left(\Omega_{1}, \Sigma_{1}, \mu\right)$, then $\left|T_{z}\left(\gamma_{z}\right)\right|$ is subharmonic for each $\omega$.

A smooth family, $T_{z}$, is of admissible growth if for all $0<r \leqslant 1$,

$$
I(z)=\int_{\Omega_{2}}\left|T_{z}\left(\gamma_{z}\right)\right|^{r}\left|\lambda_{z}\right| d \nu
$$

is of admissible growth (see Definition 1.3.1, for each analytic simple function $\lambda_{z}$ on $\left(\Omega_{2}, \Sigma_{2}, \nu\right)$ and each analytic simple function $\gamma_{z}$ on $\left(\Omega_{1}, \Sigma_{1}, \mu\right)$.

THEOREM 4.1.1. Let $T_{s}$ be a smooth family of sublinear operators of admissible growth, defined in $0 \leqslant \operatorname{Re} z \leqslant 1$. Suppose that $\varphi_{i}, Q_{i},(i=1,2)$, are $\varphi$-functions such that $\varphi_{i}(u)=\tilde{\psi_{i}}\left(u^{r_{i}}\right)$ and $Q_{i}(u)=\tilde{S_{i}^{\sim}}\left(u^{s_{i}}\right)$ where $S_{i}^{\tilde{n}}$ and $\tilde{\psi_{i}}$ are convex and $0<r_{i}, s_{i} \leqslant 1$, and let $\varphi_{t}^{-1}=\left(\varphi_{1}^{-1}\right)^{1-t}\left(\varphi_{2}^{-1}\right)^{t}$ and $Q_{t}^{-1}=\left(Q_{1}^{-1}\right)^{1-t}\left(Q_{2}^{-1}\right)^{t}$. Finally suppose

$$
\begin{gathered}
\left\|T_{i y}(f)\right\|_{r Q_{1}} \leqslant A_{1}(y)\|f\|_{r \varphi_{1}}, \\
\left\|T_{(1+i y)}(f)\right\|_{r Q_{2}} \leqslant A_{2}(y)\|r\|_{r \varphi_{2}}
\end{gathered}
$$

for each $f \epsilon L\left(\Omega_{1}, \Sigma_{1}, \mu\right)$, where $0<r \leqslant \min \left(r_{1}, r_{2}, s_{1}, s_{2}\right)$, and $\log \left|A_{i}(y)\right|$ $\leqslant A e^{a}|r|, a<\pi, i=1,2$. Then,

$$
\left\|T_{t}(f)\right\|_{r Q_{t}} \leqslant 2 A_{t}\|f\|_{r \varphi_{t}}
$$

for all $f \in L\left(\Omega_{1}, \Sigma_{1}, \mu\right)$ and where

$$
\log A_{t}=\int_{-\infty}^{\infty} \omega(1-t, y) \log \left(2 A_{1}(y)\right) d y+\int_{-\infty}^{\infty} \omega(t, y) \log \left(\left(2 A_{2}(y)\right) d y\right.
$$

and $\omega(t, y)$ is defined in section 1.

Proof. Let $\psi_{i}(u)=\tilde{\psi_{i}}\left(u^{r_{i} / r}\right), S_{i}(u)=S_{i}\left(u^{\delta_{i} / r}\right), \psi_{t}^{-1}=\left(\psi_{1}^{-1}\right)^{1-t}\left(\psi_{2}^{-1}\right)^{t}$ and $S_{t}^{-1}=\left(S_{1}^{-1}\right)^{1-t}\left(S_{2}^{-1}\right)^{t}$. Then $\varphi_{i}(u)=\psi_{i}\left(u^{r}\right), \quad Q_{i}(u)=S_{i}\left(u^{r}\right), \quad \varphi_{t}(u)$ 
$=\psi_{t}\left(u^{r}\right)$ and $Q_{t}(u)=\psi_{t}\left(u^{r}\right)$ with $\psi_{i}, \psi_{t}, Q_{i}$ and $Q_{t}$ all convex. Let $R_{1}$ and $R_{2}$ be the complementary functions to $S_{1}$ and $S_{2}$ and $R_{t}^{-1}$ $=\left(R_{1}^{-1}\right)^{1-t}\left(R_{2}^{-1}\right)^{t}$. Let $\alpha_{z}$ and $\beta_{z}$ be as in Theorem 2.1.1. Let $f \in L\left(\Omega_{1}, \Sigma_{1}, \mu\right)$ with $\|f\|_{r \varphi_{t}}=1$ and $g \epsilon L\left(\Omega_{2}, \Sigma_{2}, \mu\right)$ with $\|\dot{g}\|_{1 R_{t}} \leqslant 1$, and consider

$$
I=\int_{\Omega}\left|T_{t}(f)\right|^{r}|g| d v
$$

Suppose $f=\sum_{j=1}^{m_{1}} a_{j} \chi_{F_{j}}$ and $g=\sum_{k=1}^{m_{2}} b_{k} \chi_{G_{k}}$ and define

and

$$
F_{z}=\alpha_{z}\left(\varphi_{t}(|b|)\right) e^{i \theta}=\sum_{j=1}^{m_{1}} a_{z}\left(\varphi_{t}\left(\left|a_{j}\right|\right)\right) e^{i 0_{j}} \chi_{F_{j}}
$$

and finally,

$$
G_{z}=\beta_{z}\left(R_{t}(|g|)\right) e^{i \beta^{\prime}}=\sum_{k=1}^{m_{2}} \beta_{z}\left(R_{t}\left(\left|b_{k i}\right|\right)\right) e^{i \theta_{k}} \chi_{G_{k}}
$$

$$
\gamma_{z}^{k}=\sum_{j=1}^{m_{1}} \beta_{z}^{1 / r}\left(R_{t}\left(\left|b_{k l}\right|\right)\right) \alpha_{z}\left(\varphi_{t}\left(\left|a_{j}\right|\right)\right) e^{i\left(\theta_{j}+\theta_{k}^{\prime}\right)} \chi_{F_{j}} .
$$

Note that $\gamma_{z}^{k}$ is an analytic simple function for each $k=1,2, \ldots, m_{2}$. Consider the following extension of $I$,

$$
I(z)=\int_{\Omega_{2}}\left|T_{z}\left(F_{z}\right)\right|^{r}\left|G_{z}\right| d \nu=\sum_{k=1}^{m_{2}} \int_{\sigma_{k}}\left|T_{z}\left(\gamma_{z}^{k}\right)\right|^{r} d \nu .
$$

$I(z)$ has the following properties:

(i) $I(z) \geqslant 0, \log I(z)$ is subharmonic in $0<\operatorname{Re} z<1$, and $I(z)$ is continuous on $0 \leqslant \operatorname{Re} z \leqslant 1$

(ii) $I(z)$ is of admissible growth in $0 \leqslant \operatorname{Re} z \leqslant 1$.

These properties shown using the methods in the proof of Theorem $2.1,2$ since $T_{z}$ is a smooth family.

(iii) $I(i y) \leqslant 2 A_{1}(y)$ and $I(1+i y) \leqslant 2 A_{2}(y)$. For consider

$$
\begin{aligned}
I(i y) & =\int_{\Omega_{2}}\left|T_{i y}\left(F_{i y}\right)\right|^{r}\left|G_{i y}\right| d v \leqslant 2\left\|T_{i y}\left(F_{i y}\right)\right\|_{r_{1}}\left\|G_{i y}\right\|_{1 R_{1}} \\
& \leqslant 2 A_{1}(y)\left\|F_{i y}\right\|_{r \varphi_{1}}\left\|G_{i y}\right\|_{1 R_{1}} \leqslant 2 A_{1}(y)
\end{aligned}
$$

by construction of $F_{i y}$ and $G_{i y}$. Similarly $I(1+i y) \leqslant 2 A_{2}(y)$.

$$
\text { (iv) } I=I(t) \text {. }
$$

So by the lemma of Hirchman (Lemma 1.3.3) $I(t) \leqslant A_{t}$ where $A_{t}$ is defined in the statement of the theorem. Therefore, as before, (4.1.6)

$$
\left\|T_{t} f\right\|_{r_{t}} \leqslant 2 A_{t}\|f\|_{r \varphi_{t}}
$$

for each $f \in L\left(\Omega_{1}, \Sigma_{1}, \mu\right)$. This proves the theorem.
Remark. Defining $T_{z}(f)=k^{z} T\left(u^{-z} f\right), T_{z}$ can be shown to be a smooth family of operators using the methods of the proof of Theorem 3.1.2. In this case Theorem 3.1.2 is a corollary of Theorem 4.1.1.

4.2. Change of measures. Let the $\varphi$-function of Theorem 3.1.2 be $\varphi_{i}(u)=|u|^{p_{i}}$ and $Q_{i}(u)=|u|^{q_{i}}$ where $1<p_{i}, q_{i},<\infty$. Let $\frac{1}{p_{t}}=\frac{1-t}{p_{1}}+$ $+\frac{t}{p_{2}}$ and $\frac{1}{q_{t}}=\frac{1-t}{q_{1}}+\frac{t}{q_{2}}$. Then the theorem takes the following form.

(4.2.1) $\quad\left\|k_{i}(T f)\right\|_{q_{i, v}} \leqslant M_{i}\left\|u_{i} f\right\|_{p_{i, \mu}}, \quad f \in L^{p_{i}}, \quad i=1,2$

then

(4.2.2) $\quad\left\|k_{t}(T f)\right\|_{q_{t, \nu}} \leqslant M_{1}^{1-t} M_{2}^{t}\left\|u_{t} f\right\|_{t_{t, \mu}}, \quad f \in L^{p_{t}}$

where $\|f\|_{p, \mu}=\left(\int_{\Omega}|f|^{p} d \mu\right)^{1 / p}$. (The constant 4 can be removed in the $L^{p}$ case.)

Suppose $\mu_{1}, \mu_{2}, v_{1}$ and $\nu_{2}$ are measures with the following property. There exist positive measurable functions $\alpha_{1}, \alpha_{1}, \beta_{1}$ and $\beta_{2}$ such that

Define

and

$$
u_{i}(A)=\int_{A} a_{i} d \mu, \quad \text { all } A \epsilon \Sigma_{1},
$$

$$
v_{i}(A)=\int_{A} \beta_{i} d v, \quad \text { all } A \in \Sigma_{2} .
$$$$
\text { If } u_{i}=a_{1}^{1 / p i}, k_{i}=\beta_{i}^{1 / q i}, s(t)=\frac{t p_{t}}{p_{1}} \text { and } r(t)=\frac{t q_{t}}{q_{1}} \text {, then (see [30] for }
$$
details) equations (4.2.1) and (4.2.2) take the following form,

$$
\|T f\|_{q_{i},{ }_{i}} \leqslant M_{i}\|f\|_{p_{i}, \mu_{i}}, \quad f \in L^{p_{i}, \mu_{i}}
$$

and

$$
\|T f\|_{q_{t},{ }_{r}(t)} \leqslant M_{1}^{1-t} M_{2}^{t}\|f\|_{p_{t}, \mu_{s(t)}}
$$

which is the result proved in [30].

Now suppose the $\varphi$-functions in Theorem 2.1.2 are all convex and all satisfy the following condition

$$
\varphi(u v) \leqslant \varphi(u) \varphi(v) \quad \text { for } u, v \geqslant 0 .
$$


Also let $\mu$ and $\nu$ satisfy (4.2.3) and (4.2.4). Then Theorem. 3.1.2 can be shown to include the result of Rao [24].

At this time, it is not clear that Theorem 3.1.2 can be ised to extend the change of measures theorem further (i.e. to more general $p$-functions). The problem seems to depend on some multiplicative property of the $\varphi$-functions, as in (4.2.9).

Using Theorem 2.1.1 it is possible to prove a result on positive operators similar to ([1], Theorem 1), and then it seems an extension of the Dunford-Schwartz-Hopf ergodic theorem can be obtained.

Acknowledgment. This paper constitutes part of the authors doctoral dissertation written at Carnegie-Mellon University under the guidance of Professor M. M. Rao. The author gratefully acknowledges Dr. Rao's interest and many helpful suggestions.

\section{Bibliography}

[1] M. A. Akcoglu and R. V. Chacon, A convexity theorem for positive operators, Z. Wahrscheilichkeitstheorie, 3 (1965), pp. 328-332.

[2] N. Aronszajn and E. Gagliardo, Interpolation spaces and interpolation methods, Ann. Math. Pura Appl., 68 (1965), pp. 51-118.

[3] P. L. Butzer and H. Berens, Semi-Groups of Operators and Approximation, New York 1967.

[4] A. P. Calderón, Intermediate spaces and interpolation, the complex method, Studia Math., 24 (1964), pp. 113-190.

[5] - and A. Zygmund, On the theorem of Hausdorff-Young and its extensions, Annals of Math. Studies, 25 (1950), pp. 166-188.

[6] - A note on the interpolation of sublinear operations, Amer. J. Math. 78 (1956), pp. 282-288.

[7] N. Dinculeanu, Espaces d'Orticz de champs de vecteurs, Rendiconti Accad. Naz. Lincei, 22 (1957), pp. 269-275.

[8] N. Dunford and J. T. Schwartz, Linear Operators: Part I, Now York 1958.

[9] P. Halmos, Measure Theory, New York 1950.

[10] E. Hewitt and K. S tromberg, Real and Abstract Analysis, Now York 1965.

11] I. I. Hirschman, Jr., A convexity theorem for certain groups of transformations, J. d'Analyse Math., 2 (1953), pp. 209-218.

[12] - A maximal problem in harmonic analysis-II, Pacific J. Math., 9 (1959), pp. 525-540.

[13] K. Hoffman, Banach Spaces of Analytio Functions, Now York 1962.

[14] M. A. Krasnosel'skii and Ya. B. Rutickii, Oonvex Functions and Orliow Spaces, (Translation), Groningen 1961.

[15] W. T. Kraynek, Interpolation of Multilinear Funotionals on $I^{\text {pw }}$ spaoes, J. Math. Anal. and Appl. 31 (1970), pp. 418 430.

[16] R. Lésniewicz, On Hardy-Orticz spaces, I, Bull. Acad. Polon., Ser: sci. math., astronom. et phys., 14 (1966), pp: 145-150.

[17] J. L. Lions, Sur les espaces d’interpolation; dualitó, Math. Skand., 9 (1961), pp. 147-177.

[18] W. Matuszewska, On generalized Orlicz spaces, Bull. Acad. Polon. Sci., Ser. soi. math., astronom. et phys., 8 (1960), pp. 349-353.

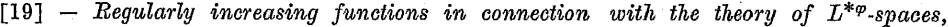
Studia Math., 21 (1962), pp. 317-344.

[20] J. Marcinkiewicz, Sur l'interpolation d'operations, C. R., Acad. Sci. Paris 208 (1939), pp. 1272-1273.

[21] G. O. Okikiolu, A convexity theorem for multitinear functionals. II, J. Math. Anal. Appl., 16 (1966), pp. 396-404.

[22] J. Peetre, A Theory of Interpolation of Normed Spaces, Notes Universidade de Brasilia, (1963).

[23] T. Rado, Subharmonic Functions, Chelsea, New York 1949

[24] M. M. R ao, Interpolation, ergodicity, and martingales, J. Math. Mech., 16 (1960), pp. 543-567.

[25] - Extensions of the Hausdorff-Young Theorem, Israel J Math. July 1968

[26] - Smoothness of Orlicz spaces, Indag. Math., 27 (1965), pp. 670-690.

[27] M. Riesz, Sur les maxima des formes bilineaires et sur les functionnelles lineaires, Acta. Math., 49 (1926), pp. 465-497.

[28] W. J. Riordan, Unpublished Ph. D. thesis, University of Chicago, (1957).

[29] E. M. Stein, Interpolation of linear operators, Trans. Amer. Math. Soc., 83 (1956), pp. $482-492$.

[30] - and G. Weiss, Interpolation of operators with change of measures, Trans. Am. Math. "Soc., 87 (1958), pp. 159-172.

[31] G. Weiss, A note on Orlicz spaces, Portugaliae Mat., 15 (1956), pp. 35-47.

[32] - An interpolation theorem for sublinear operations on $H^{p}$ spaces, Proc. Am. Math. Soc., 8 (1957), pp. 92-99.

[33] A. C. Zaanen, Linear Analysis, Amsterdam 1953.

[34] A. Zygmund, Trigonometric Series, Vols. I, II., Cambridge 1959.

COMPUTER SCIENCE DEPARTMENT

UNIVERSITY OF PITTSBURGE

Received May 16, 1971 